\title{
Geometric gauge potentials and forces in low-dimensional scattering systems
}

\author{
B. Zygelman* ${ }^{*}$ \\ Department of Physics and Astronomy, University of Nevada, Las Vegas, Las Vegas NV 89154
}

(Dated: May 1, 2022)

\begin{abstract}
We introduce and analyze several low-dimensional scattering systems that exhibit geometric phase phenomena. The systems are fully solvable and we compare accurate solutions of them with those obtained in a Born-Oppenheimer projection approximation. We illustrate how geometric magnetism manifests in them, and explore the relationship between solutions obtained in the diabatic and adiabatic pictures. We provide an example, involving a neutral atom dressed by an external field, in which the system mimics the behavior of a charged particle that interacts with, and is scattered by, a ferromagnetic material. We also introduce a similar system that exhibits Aharonov-Bohm scattering. We propose possible practical applications. We provide a theoretical approach that underscores universality in the appearance of geometric gauge forces. We do not insist on degeneracies in the adiabatic Hamiltonian, and we posit that the emergence of geometric gauge forces is a consequence of symmetry breaking in the latter.
\end{abstract}

PACS numbers: 03.65.-w,03.65.Aa,03.65Nk,03.65.Vf,34.30.Cf

\section{INTRODUCTION}

The Born-Oppenheimer (BO) approximation allows the replacement of a complex many-body problem by a mean field description in which the motion of atoms can be accurately described. The dynamics of the latter are governed by an effective force $\boldsymbol{F}$ generated by a scalar potential that represents the collective motion of the fast degrees of freedom, i.e. the electrons. According to the Hellmann-Feynman theorem [1]

$$
\boldsymbol{F}=-\nabla \epsilon(\boldsymbol{R})
$$

where $\epsilon$ is the Born-Oppenheimer energy (including higher-order corrections [2]) of the electronic state and $\boldsymbol{R}$ typically describes the geometry of the atomic ensemble. Molecular spectroscopy, atomic collision phenomena, as well as molecular dynamics simulations are just some of the applications that derive their utility from the implications of Eq. (1). In its modern expression [3 [5] BO theory also allows for an additional contribution to Eq. (1), that of an induced effective "magnetic" force 5 - 8

$$
\begin{aligned}
& \boldsymbol{F}_{m} \equiv \frac{\boldsymbol{v}}{2} \times \boldsymbol{B}-\boldsymbol{B} \times \frac{\boldsymbol{v}}{2} \\
& \boldsymbol{B}=\boldsymbol{\nabla} \times \hbar \boldsymbol{A}
\end{aligned}
$$

where $\boldsymbol{v}$ is the atom velocity operator [5] and $\boldsymbol{A}$ is a vector potential. This effective force is sometimes called geometric magnetism, a term coined by M.V. Berry, and is the nomenclature we adopt here.

Advances 9 14 in the manipulation of cold atomic matter has allowed researchers to "dress" atoms via the application of laser fields in order to induce gauge potentials that manifest as effective Lorentz forces on the atoms. This phenomenon has been called, "artificial" 13

\footnotetext{
*bernard@physics.unlv.edu
}

or "synthetic" 10 magnetism and in a laboratory realization of it [9], a BEC cloud was observed to undergo cyclotron motion in the same manner charged particles behave in a magnetic field. The effect has important implications for the control of atomic matter, and offers a novel route to quantum magnetism [12, 15].

In this paper we address the question; what is the relationship between geometric and induced synthetic magnetism? The appearance of vector gauge potentials in molecular physics was first demonstrated by Mead and Truhlar [16] in their study of pathologies associated with conical intersections (degeneracies of distinct BO molecular energies at an isolated point $\boldsymbol{R}_{c}$ ). At a conical intersection the phase of the $\mathrm{BO}$ wavefunction is undefined and, in order to avoid multi-valuedness, Mead and Truhlar introduced a compensating phase factor to accompany the nuclear wavefunction. They argued that such a procedure is equivalent to minimally coupling the nuclear motion with a non-trivial vector potential[16. The Mead-Truhlar gauge potential does not exhibit geometric magnetism but since Berry's vector potential [6, 17] has non-vanishing curl, systems exhibiting the latter do. In the Mead-Truhlar treatment degeneracy plays a central role as it does in Berry's Hamiltonian[17. Degeneracy is also an essential ingredient in the non-Abelian generalization of Berry's phase 18 . Thus the appearance of geometric phases has historically been associated with systems in which degeneracies manifest. This does not appear to be the case for artificial magnetism[13 and so it is worthwhile to further investigate the relationship between the two phenomena.

In applications 19 21 it is often necessary to go beyond BO theory and exploit a multi-channel generalization of it, the Born-Huang expansion or the method of perturbed stationary states (PSS) 22. In atomic collision theory the latter lead to equations that have the form $[5]$

$$
-\frac{\hbar^{2}}{2 m}(\boldsymbol{\nabla}-i \boldsymbol{A})^{2} F+V F=E F
$$

where $F$ is a multi-channel amplitude expressed as a col- 
umn matrix, $\boldsymbol{A}$ is a matrix-valued vector potential, and $V$ is a diagonal scalar-valued matrix whose elements consist of the BO eigenvalues associated with each channel and $E$ is the total energy eigenvalue. If a complete set of channel states are included in the PSS expansion then Eq. (3) is exact. We distinguish two cases in applications of Eq. (3). In the first, matrix $V$ is degenerate at all $\boldsymbol{R}$ and a gauge transformation can be found [5, 7] so that, in the new gauge, $\boldsymbol{A}=0$. The representation in which $\boldsymbol{A}=0$ is sometimes called the diabatic picture [5, 23, whereas Eq. (3) describes dynamics in the adiabatic picture. The second case, which we deal with exclusively in this paper, $V$ is non-degenerate and Eq. (3) exhibits gauge covariance if the vector valued potential $\boldsymbol{A}$ is taken as the spatial component of a $3+1$ gauge field that also contains a temporal component [5]. In applications 19 21] one typically truncates the PSS equations to a finite number of channels (most realistic systems involve an infinite number of channels) and in that case one cannot, in general, find a transformation into a gauge where $\boldsymbol{A}=0$. As a consequence effective gauge forces [5, 19] manifest. Does this imply that the existence of the latter are the artifacts of an approximation procedure? What is the relationship between geometric forces and the fictitious forces that arise in a classical description where non-inertial frames are employed?

In order to address these, and other questions, we introduce and analyze several low dimensional but solvable models that exhibit geometric phase phenomena in the BO approximation. In the systems discussed here the "fast" degrees of freedom span a finite Hilbert space of dimensions $d=2,4$. Similar models have been discussed previously 24-26 but here we focus on systems that do not exhibit energy degeneracies in the adiabatic approximation. We concentrate our efforts on fully quantal treatments of Eq. (3) and we limit our discussion to scattering systems and their solution.

In section II we provide a general theoretical framework for the subsequent discussions. We introduce a 1D scattering system in which geometric phase phenomena arise and which fully quantal analytic solutions are available. In section III we introduce a $2 \mathrm{D}$ scattering system that exhibits the phenomenon of synthetic, or geometric, magnetism. We compare the scattering solutions of it with that obtained for a charged particle scattered by a ferromagnetic slab. In the remaining sections we show how these systems are related to ones in which geometric forces arise due to inter-particle interactions. An example involving two, interacting, spin-1/2 particles is presented.

Vector valued quantities will be shown in boldface, we will announce matrix-valued quantities in the text and use ordinary Roman typeface to represent them. However, in certain circumstance, where there might be a possibility for confusion, we will underline a Roman letter to stress its matrix nature.
II.

\section{A. General Theory}

Consider the Hamiltonian

$$
H=-\frac{\hbar^{2}}{2 m} \nabla_{\boldsymbol{R}}^{2}+H_{a d}(\boldsymbol{R})
$$

where $H_{a d}(\boldsymbol{R})$ is the Hamiltonian defined on an $n$ dimensional Hilbert space $h_{n}$ that represents the internal, or "fast" [5], degrees of freedom of a quantum system of mass $m$. It is parameterized by the eigenvalues of the quantum variable $\boldsymbol{R}$. In molecular physics $H_{a d}$ is called the adiabatic Hamiltonian and represents the total kinetic, electrostatic, and magnetic interactions among the electrons. The kinetic energy term in Eq. (4) represents the motion of the "slow" degrees of freedom. In molecular physics the internal space $h_{n}$ is spanned by the BornOppenheimer (BO) eigenstates of $H_{a d}$. Additional realizations described by Eq. (4) could be an atom, molecule or spin- $n$ system in an external field that is modulated by the values of $\boldsymbol{R}$. If we assume that the basis for $h_{n}$ is finite, we can express

$$
H_{a d}(\boldsymbol{R})=U(\boldsymbol{R}) H_{B O} U^{\dagger}(\boldsymbol{R})
$$

where $H_{B O}$ is a diagonal matrix whose entries we shall label $e_{1}(\boldsymbol{R}), e_{2}(\boldsymbol{R}), \ldots e_{n}(\boldsymbol{R})$. In molecular physics they are called the BO eigenvalues for the electronic Hamiltonian $H_{a d} . U(\boldsymbol{R})$ is a unitary operator acting on the internal states and, in general, is also parameterized by $\boldsymbol{R}$. Expressed as a matrix representation in the BO basis, $U$ is an $n \times n$ unitary matrix.

Any well behaved Hermitian operator $H_{a d}$ can be written in the form given by Eq. (5), however we require the additional condition that $U$ must be single-valued in the parameter space spanned by $\boldsymbol{R}$. Consider the unitary matrix,

$$
W^{\dagger}(\boldsymbol{R})=\mathcal{P} \exp \left(i \int_{\mathcal{C}}^{\boldsymbol{R}} d \boldsymbol{R}^{\prime} \cdot \boldsymbol{A}\left(\boldsymbol{R}^{\prime}\right)\right) C
$$

where $\boldsymbol{A}$ is a vector valued $n \times n$ matrix (i.e. a nonAbelian gauge potential), $\mathcal{P}$ represents a path-ordering operator and $C$ is a constant unitary matrix. Pathordered integrals along curve $\mathcal{C}$ in parameter space $\boldsymbol{R}$ are defined as follows: Consider a path, $\mathcal{C}$, defined by the set $\boldsymbol{R}_{1}, \boldsymbol{R}_{2}, \ldots \boldsymbol{R}_{n}$ where $\boldsymbol{R}_{1}=\boldsymbol{R}\left(t_{1}\right), \boldsymbol{R}_{2}=\boldsymbol{R}\left(t_{2}\right) \ldots \boldsymbol{R}_{n}=$ $\boldsymbol{R}\left(t_{n}\right), \boldsymbol{R}_{n}=\boldsymbol{R}$, and $t_{1}<t_{2}<\ldots t_{n}$ defines a trajectory that maps out the path. We define

$$
\begin{aligned}
& \mathcal{P} \exp \left(i \int_{\mathcal{C}}^{\boldsymbol{R}} d \boldsymbol{R}^{\prime} \cdot \boldsymbol{A}\left(\boldsymbol{R}^{\prime}\right)\right) \equiv \\
& T \exp \left(i \int_{t_{1}}^{t_{n}} d t \frac{d \boldsymbol{R}(t)}{d t} \cdot \boldsymbol{A}(t)\right)
\end{aligned}
$$

where we assumed that the path is sufficiently smooth so that $\frac{d \boldsymbol{R}(t)}{d t}$ is well defined on it and $T$ is the Dyson timeordering operator. For open ended paths, such integrals 
are also called the Wilson-line 27, and if $\mathcal{C}$ traces a closed path Eq. (6) represents the Wilson loop integral[28] which we require, for a pure gauge, to have the value of unity. To that end, we demand that

$$
F_{\mu \nu}=\partial_{u} A_{\nu}-\partial_{\nu} A_{\mu}-i\left[A_{\mu}, A_{\nu}\right]=0
$$

where we have used the notation defined in [5] and, here, $\mu, \nu$ are spatial indices only. We make the assumption, if $\boldsymbol{A}$ satisfies condition Eq. (8) and is not singular, then the value for path integral Eq. 6 is independent of $\mathcal{C}$. With this working assumption, we take the gradient of Eq. (6)

$$
\begin{aligned}
& \boldsymbol{\nabla} W^{\dagger}=i \boldsymbol{A} W^{\dagger} \\
& \left(\boldsymbol{\nabla} W^{\dagger}\right) W=i \boldsymbol{A}
\end{aligned}
$$

or

$$
\left(\nabla W^{\dagger}\right) W=-W^{\dagger} \nabla W=i \boldsymbol{A}
$$

and

$$
\boldsymbol{A}=i W^{\dagger} \nabla W
$$

Consider a differentiable unitary operator $U(\boldsymbol{R})$ so that $i U^{\dagger} \nabla U=\boldsymbol{A}$, and therefore satisfies Eq. (8) [7, 39]. Since $U$ and $W$ are both unitary and differentiable, $W=$ $U Z$ where $Z=U^{-1} W$. Inserting the expression for $W$ into the r.h.s. of Eq. 111 we obtain

$$
\begin{aligned}
& i W^{\dagger} \boldsymbol{\nabla} W=\boldsymbol{A}=Z^{\dagger} \boldsymbol{A} Z+i Z^{\dagger} \boldsymbol{\nabla} Z \text { or } \\
& {[Z, \boldsymbol{A}]=i \boldsymbol{\nabla} Z .}
\end{aligned}
$$

The unitary matrix $Z$ that relates $U$ with $W$ must obey the above constraint equation and represents a nonAbelian gauge transformation in which $\boldsymbol{A}$ remains invariant (e.g. for the Abelian case it requires that $\mathrm{Z}$ is constant). If $W$ and $U$ differ, they do so up to an inconsequential gauge transformation. For example, if we replace $U$ in the adiabatic Hamiltonian, given in Eq. (5), with $W$ then Hamiltonian Eq. (4) is replaced with

$$
H^{\prime}=-\frac{\hbar^{2}}{2 m} \nabla_{\boldsymbol{R}}^{2}+W H_{B O} W^{\dagger} .
$$

It will follow from the discussion below that both $H$, and $H^{\prime}$ lead to identical Schrodinger equations in the adiabatic gauge since both formulations share the same vector and scalar gauge potentials. Therefore, we can always replace the adiabatic Hamiltonian with one in which $U$ is parameterized by a Wilson line as given by Eq. (6).

\section{B. Illustrative Example}

As an illustration, consider the direct product of an $h_{n}=2$ dimensional Hilbert space of a two-level, or qubit, system and a 1D Hilbert space for quantum variable $\boldsymbol{R}$, e.g. $\quad \boldsymbol{R}$ is represented by the one-dimensional variable $-\infty<x<\infty$. We define the gauge field

$$
\boldsymbol{A}=\left(\begin{array}{cc}
A_{0} & A_{1} \\
A_{1} & -A_{0}
\end{array}\right)
$$

where $A_{0}, A_{1}$ are real constants. It is evident that condition Eq. (8) is satisfied by this gauge potential and we can apply Eq. (6) to construct

$$
\begin{aligned}
& U=\left(\begin{array}{cc}
\cos (A x)-\frac{i A_{0} \sin A x}{A} & -\frac{i A_{1} \sin A x}{A} \\
-\frac{i A_{1} \sin A x}{A} & \cos (A x)+\frac{i A_{0} \sin (A x)}{A}
\end{array}\right) \\
& A \equiv \sqrt{A_{1}{ }^{2}+A_{0}{ }^{2}} .
\end{aligned}
$$

We note that $U$ is single-valued and $U(x=0)$ is the identity operator.

Suppose the internal Hamiltonian is given by

$$
H_{a d}(x)=U_{0} H_{B O} U_{0}^{\dagger}
$$

where $H_{B O}$ is an arbitrary diagonal matrix. Previous studies e.g. [16, 24 have focused on systems in which $H_{B O}$ is degenerate for some value of the parameter $\boldsymbol{R}_{c}$, i.e. $e_{1}\left(\boldsymbol{R}_{c}\right)=e_{2}\left(\boldsymbol{R}_{c}\right)$. Here we do not allow any crossings, indeed we take the $\mathrm{BO}$ eigenergies to be constant throughout the domain of $x$. We define

$$
\begin{aligned}
& U_{0}=\left(\begin{array}{ll}
1 & 0 \\
0 & 1
\end{array}\right) \quad x<0 \\
& U_{0}=U \quad x>0
\end{aligned}
$$

where $U$ is given by Eq. 13 . $U_{0}$ is continous in $x$ but not differentiable at $x=0$. Choosing

$$
H_{B O}=\left(\begin{array}{cc}
\Delta & 0 \\
0 & -\Delta
\end{array}\right),
$$

where $\Delta>0$ is a constant, we obtain the set of coupled Schrodinger equations for the spinor eigenstates of Hamiltonian (4)

$$
\underline{F}^{\prime \prime}-\frac{2 m}{\hbar^{2}} V \underline{F}+\frac{2 m}{\hbar^{2}} E \underline{F}=0
$$

where

$$
\underline{F} \equiv\left(\begin{array}{c}
F_{c}(x) \\
F_{o}(x)
\end{array}\right)
$$

and

$$
\begin{aligned}
& V=H_{B O}=\left(\begin{array}{cc}
\Delta & 0 \\
0 & -\Delta
\end{array}\right) \quad x<0 \\
& V=U H_{B O} U^{\dagger} \quad x \geq 0 .
\end{aligned}
$$

We seek scattering solutions to Eq. 177 and if we set $E=-\Delta+\frac{\hbar^{2} k^{2}}{2 m}$ where $\frac{\hbar^{2} k^{2}}{2 m}<2 \Delta$, the excited internal $\mathrm{BO}$ channel is closed.

Because $H_{B O}$ is diagonal and constant, instead of solving Eqs. 17) directly it is convenient to transform to the adiabatic picture whose wave function is,

$$
\underline{F}_{a d}=U_{0}^{\dagger} \underline{F}
$$

and satisfies $[5]$

$$
(\nabla-i A)^{2} \underline{F}_{a d}-\frac{2 m}{\hbar^{2}} H_{B O} \underline{F}_{a d}+\frac{2 m}{\hbar^{2}} E \underline{F}_{a d}=0
$$


where $A$ is the gauge potential

$$
\begin{aligned}
& A=\left(\begin{array}{cc}
A_{0} & A_{1} \\
A_{1} & -A_{0}
\end{array}\right) \quad x>0 \\
& A=0 \quad x<0 .
\end{aligned}
$$

In the adiabatic picture we have replaced the off-diagonal potential matrix $V$ with the diagonal $\mathrm{BO}$ matrix, for the price of gauge potentials. At collision energies where some of the excited BO channels are closed, a common approximation in molecular and collision physics is to project the system of coupled equations (which are exact) onto the open sector. This is called the BornOppenheimer $(\mathrm{BO})$ or, if several channels are open, the perturbed stationary states (PSS) approximation [5, 22. In our example this approximation leads to the following single channel equation [5],

$$
\begin{aligned}
& \left(\partial+i A_{0}\right)^{2} F_{o}+k^{2} F_{o}-b F_{o}=0 \quad x \geq 0 \\
& \partial^{2} F_{o}+k^{2} F_{o}=0 \quad x<0 \\
& b \equiv \sum_{k \neq i} \boldsymbol{A}_{i k} \cdot \boldsymbol{A}_{k i}=A_{1}^{2}
\end{aligned}
$$

The induced potential $b$ is closely related to the so-called "B" term, or adiabatic, correction 2, 29, 30. A gauge transformation $F_{o} \rightarrow \exp \left(-i A_{0} x\right) F_{o}$ allows us to "eliminate" the derivative coupling in Eq. (23) and we can proceed to solve the scattering problem. Imposing the boundary condition that the open-channel wavefunction vanishes at $x=L>>0$ (which is equivalent to the placement of an impenetrable barrier at $L$ ), we obtain, in the asymptotic region for $x<0$,

$$
\begin{aligned}
& F_{0}(x)=\exp (i k(x-L))+R \exp (-i k(x-L)) \\
& R=\exp (-2 i k L) \times \\
& \left(-1+\frac{2 k}{k+i \sqrt{k^{2}-A_{1}^{2}} \cot \left(L \sqrt{k^{2}-A_{1}^{2}}\right)}\right) \quad k>\left|A_{1}\right| \\
& \text { and for } k<\left|A_{1}\right| \\
& R=\exp (-2 i k L) \times \\
& \left(-1+\frac{2 k}{k+i \sqrt{A_{1}^{2}-k^{2}} \operatorname{coth}\left(L \sqrt{A_{1}^{2}-k^{2}}\right)}\right) .
\end{aligned}
$$

We can also solve the fully coupled Eqs. 21 analytically, as outlined in the appendix, without resorting to the Born-Oppenheimer approximation. That solution, in the adiabatic limit, leads to

$$
R=-1+2 i k\left(L-\frac{\tanh \left(A_{1} L\right)}{A_{1}}\right)+\mathcal{O}\left(k^{2}\right)+\ldots
$$

where we have kept only the lowest order terms, as $k \rightarrow 0$, in an effective range expansion for $R$. This result is in harmony with that obtained in an effective range expansion of expression (24) obtained using the BO approximation.

In summary,
- At collision energies where the excited internal state is closed and the energy defect between the open and closed channels is large, the lowest order term in an effective range expansion (i.e. in the cold collision energy regime) of the Born-Oppenheimer expression for the reflection coefficient is identical to that obtained by solution of the fully coupled equations. It depends on the value of the offdiagonal component of the induced gauge potential (12) despite the fact that the pure gauge condition Eq. (8) is satisfied.

- In the BO approximation the off-diagonal gauge coupling leads to a higher order induced scalar potential, given by the expression for $b$ in Eq. 23. In this approximation the diagonal component $A_{0}$ can simply be "gauged" away and physical quantities, such as the scattering length, are independent of it.

- At collision regimes where the BO approximation is no longer appropriate 31, (e.g. when $\Delta \sim E$ ), the diagonal components $A_{0}$ do affect scattering properties. This may seem counter-intuitive since we stated that a gauge transformation can be employed to transform these potentials away. One way of proceeding beyond BO theory is to describe the coupling between the open and closed channel through the introduction of a non-local optical potential 32. In such a treatment Eq. 23] is replaced by 33

$\left(\partial+i A_{0}\right)^{2} F_{o}+k^{2} F_{o}+\frac{2 m}{\hbar^{2}} V_{o p t} F_{o}=0 \quad x \geq 0$

$V_{o p t} F_{o} \equiv \int d x^{\prime} V_{o p t}\left(x, x^{\prime}\right) F_{o}\left(x^{\prime}\right)$

where $V_{\text {opt }}$ is a nonlocal potential. Now the transformation $F_{o} \rightarrow \exp \left(-i A_{0} x\right) F_{o}$ does eliminate the derivative coupling in Eq. 26 but, since $V_{o p t}$ is non-local, it results in

$$
\begin{aligned}
& \int d x^{\prime} V_{o p t}\left(x, x^{\prime}\right) F_{o}\left(x^{\prime}\right) \rightarrow \\
& \int d x^{\prime} V_{o p t}\left(x, x^{\prime}\right) \exp \left(-i A_{0} x^{\prime}\right) F_{o}\left(x^{\prime}\right)
\end{aligned}
$$

and it cannot simply be "gauged" away as in the BO approximation.

- If the collision energy is sufficiently large so that $E>>\Delta$ and both channels are open the resulting scattering properties become independent of both $A_{0}$ and $A_{1}$. In the case where the internal state energy defect $\Delta$ can be neglected, we can treat $\underline{A}$ as a pure gauge, i.e. it does not affect the scattering properties of the system. 


\section{GEOMETRIC MAGNETISM}

The model introduced above has interesting and salient properties but it lacks the feature of geometric magnetism. Because the diagonal components $A_{0}$ can be gauged away in the adiabatic, or $\mathrm{BO}$, limit effective Lorentz forces do not manifest. Can we construct a gauge potential (again here we limit ourselves to the $h_{2}$ ) that satisfies the integrability condition Eq. (8), but includes diagonal components that are non-trivial? The answer is yes but we need to extend our parameter space, at the least, into two dimensions i.e $\boldsymbol{R}=(x, y)$, $-\infty<x, y<\infty$. Consider

$$
U=\exp \left(-i \sigma_{3} \phi(x, y)\right) \exp \left(-i \sigma_{2} \Omega(x, y)\right) \exp \left(i \sigma_{3} \phi(x, y)\right)
$$

here $\sigma_{i}$ are the Pauli spin matrices and $\phi(x, y)$ and $\Omega(x, y)$ are arbitrary but single-valued functions in the $x y$-plane.
(8). Using prescription 11 we find

\section{A. Singular model}

By construction $U$ is single valued and the gauge potentials derived from it satisfy integrability condition Eq.

$$
\boldsymbol{A}=\left(\begin{array}{cc}
-2 \sin ^{2}(\Omega) \boldsymbol{\nabla} \phi & -\exp (-2 i \phi)(\sin (2 \Omega) \boldsymbol{\nabla} \phi+i \boldsymbol{\nabla} \Omega) \\
\exp (2 i \phi)(i \boldsymbol{\nabla} \Omega-\sin (2 \Omega) \nabla \phi) & 2 \sin ^{2}(\Omega) \boldsymbol{\nabla} \phi
\end{array}\right)
$$

There is freedom of choice in $\Omega, \phi$, as long as singlevaluedness is enforced, but here let's introduce

$$
\begin{aligned}
& \phi(x, y)=L B_{0} y / 2 \\
& \Omega(x, y)= \\
& \theta_{H}(x) \theta_{H}(L-x) \operatorname{Arcsin}(\sqrt{x / L})+\theta_{H}(x-L) \frac{\pi}{2}
\end{aligned}
$$

$$
\boldsymbol{A}=\left(\begin{array}{c}
-x B_{0} \hat{\boldsymbol{j}} \\
e^{2 i \phi}\left(-\sin (2 \Omega) \frac{L B_{0}}{2} \hat{\boldsymbol{j}}+\hat{\mathbf{i}} \frac{i}{2 \sqrt{x(L-x)}}\right)
\end{array}\right.
$$

and for $x>L$

$$
\boldsymbol{A}=\hat{\boldsymbol{j}}\left(\begin{array}{cc}
-B_{0} L & 0 \\
0 & B_{0} L
\end{array}\right) .
$$

We keep $H_{B O}$ described in the previous paragraphs. In seeking scattering solutions for this system it is again convenient to proceed in the adiabatic picture. The adiabatic amplitudes obey equations (21) except that the gauge potentials are now given by Eq. (28). As above, the ground internal BO state is open and the excited state is closed and it is appropriate, for sufficiently low collision energies and a large energy gap $\Delta$, to proceed with a BO projection of Eqs. 21 into a single channel
(A similar but not equivalent system was independently proposed in Ref. 15]) where $\theta_{H}$ is the Heaviside step function and $B_{0}, L$ are positive constants. Inserting Eq. 29 into Eq. 28 we obtain, For $x<L, \boldsymbol{A}=0$, in the region $0<x<L$

$$
\left.\begin{array}{c}
\left.-e^{-2 i \phi}\left(\sin (2 \Omega) \frac{L B_{0}}{2} \hat{\boldsymbol{j}}+\hat{\mathbf{i}} \frac{i}{2 \sqrt{x(L-x)}}\right)\right) \\
x B_{0} \hat{\boldsymbol{j}}
\end{array}\right)
$$

$$
\begin{aligned}
& \partial_{x}^{2} F_{o}+\left(\partial_{y}-i A_{0}\right)^{2} F_{o}+k^{2} F_{o}-b(x) F_{o}=0 \\
& A_{0}=B_{0} x \\
& b(x)=\sum_{k \neq i} \boldsymbol{A}_{i k} \cdot \boldsymbol{A}_{k i}=\left[\sin ^{2}(2 \Omega) \nabla \phi \cdot \nabla \phi+\nabla \Omega \cdot \nabla \Omega\right]= \\
& =x(L-x) B_{0}^{2}+\frac{1}{4 x(L-x)} .
\end{aligned}
$$

This Schrodinger equation is equivalent to that obtained for a system in which a charged particle interacts with 
an effective magnetic field

$$
\boldsymbol{B}=\boldsymbol{\nabla} \times \hbar \boldsymbol{A}_{0}=\hat{\boldsymbol{k}} \hbar B_{0} \quad 0<x<L,
$$

in addition to an induced scalar potential $b(x)$. The proposed spin $1 / 2$ system does not have an electromagnetic charge and so this effective magnetic field is a manifestation of what is, sometimes, called artificial or synthetic magnetism [9 13, 34. It leads to effective Lorentz type forces acting on the dynamical system. The emergence of such forces was first demonstrated in molecular physics [5]7].

\section{B. Non-singular model}

Whereas the above model describes quantum magnetism the induced scalar potential suffer singularities at $x=0$ and at $x=L$ and so we wish to introduce a similar model that does not posses these singularities. We now posit that

$$
\begin{aligned}
& \phi(x, y)=L B_{0} y / 2 \\
& \Omega(x, y)=\frac{\pi}{4}(1+\tanh [\beta x]) .
\end{aligned}
$$

$$
A_{y}=\left(\begin{array}{cc}
-B_{0} L \sin ^{2}\left(\frac{1}{4} \pi(\tanh (\beta x)+1)\right) & -\frac{B_{0} L}{2} e^{-i B_{0} L y} \cos \left(\frac{1}{2} \pi \tanh (\beta x)\right) \\
-\frac{B_{0} L}{2} e^{i B_{0} L y} \cos \left(\frac{1}{2} \pi \tanh (\beta x)\right) & B_{0} L \sin ^{2}\left(\frac{1}{4} \pi(\tanh (\beta x)+1)\right)
\end{array}\right)
$$

and $A_{x}$ is

$$
\left(\begin{array}{cc}
0 & -\frac{1}{4} i e^{-i B_{0} L y} \pi \beta \operatorname{sech}^{2}(x \beta) \\
\frac{1}{4} i e^{i B_{0} L y} \pi \beta \operatorname{sech}^{2}(x \beta) & 0
\end{array}\right) .
$$

In the limit $x \rightarrow \infty$,

$$
A_{y} \rightarrow\left(\begin{array}{cc}
-B_{0} L & 0 \\
0 & B_{0} L
\end{array}\right)
$$

whereas $A_{x} \rightarrow 0$, and $\boldsymbol{A} \rightarrow 0$ as $x \rightarrow-\infty$.

The diagonal components of the "magnetic" induction $|\boldsymbol{B}| \equiv \hbar \frac{\partial A_{y}}{\partial x}$ are given by

$$
B(x)=\mp \frac{\hbar}{4} \pi \beta B_{0} L \operatorname{sech}^{2}(\beta x) \cos \left(\frac{\pi}{2} \tanh (\beta x)\right)
$$

where the total magnetic flux density is

$$
\Phi \equiv \int_{-\infty}^{\infty} d x \frac{|B(x)|}{\hbar}=\left|B_{0}\right| L
$$

In the $\mathrm{BO}$ approximation, for the open channel, we obtain

$$
\begin{aligned}
& \partial_{x}^{2} F_{o}+\left(\partial_{y}-i A_{0}\right)^{2} F_{o}+k^{2} F_{o}-b(x) F_{o}=0 \\
& A_{0}(x)=\Phi \sin ^{2}\left(\frac{1}{4} \pi(\tanh (x \beta)+1)\right) \\
& b(x)=\sum_{k \neq i} \boldsymbol{A}_{i k} \cdot \boldsymbol{A}_{k i}=\sin ^{2}(2 \Omega) \boldsymbol{\nabla} \phi \cdot \nabla \phi+\nabla \Omega \cdot \nabla \Omega= \\
& \frac{1}{16}\left(2 \Phi^{2}(\cos (\pi \tanh (\beta x))+1)+\pi^{2} \beta^{2} \operatorname{sech}^{4}(\beta x)\right) .
\end{aligned}
$$

We then obtain for the vector potential, $\boldsymbol{A}=A_{x} \hat{\boldsymbol{i}}+$ $A_{y} \hat{\boldsymbol{j}}$, where

We assume the incoming wave is incident in the normal direction, i.e. $F_{o}=F_{o}(x)$ and the effective equation for the $\mathrm{BO}$ amplitude is,

$$
\partial_{x}^{2} F_{o}-A_{0}^{2}(x) F_{o}+k^{2} F_{o}-b(x) F_{o}=0,
$$

or

$$
\begin{aligned}
& \partial_{x}^{2} F_{o}+k^{2} F_{0}-v_{e f f}(x) F_{o}=0 \\
& v_{e f f}(x)=A_{0}^{2}(x)+ \\
& \frac{1}{16}\left(2 \Phi^{2}(\cos (\pi \tanh (\beta x))+1)+\pi^{2} \beta^{2} \operatorname{sech}^{4}(\beta x)\right)
\end{aligned}
$$

In Figure 1 we plot the effective potential for a given $\beta$ and $\Phi \equiv B_{0} L$. We note that transmission occurs when $k^{2}$ is greater than the height of the potential barrier $\Phi^{2}$ as $x \rightarrow \infty$. In that diagram we superimpose the effective potential discussed in the appendix for the case of a ferromagnetic slab characterized by the same value of $\Phi$. In the classical description the value $\Phi$ determines whether the particle has sufficient velocity so that its Larmor radius is greater than the slab thickness and can thus penetrate the slab. Since $\boldsymbol{A} \rightarrow 0$, as $x \rightarrow-\infty$ we impose the incoming boundary condition

$$
F_{0}(x) \rightarrow \exp (i k x)+r \exp (-i k x)
$$

where $r$ is the reflection coefficient. If $k>\Phi$ transmission into the asymptotic region $x \rightarrow \infty$ is allowed and, in this region, the vector potential has the form given by Eq. (37). The outgoing asymptotic current is given by (see appendix A)

$$
\begin{aligned}
& j_{x}=\frac{\hbar}{m}|t|^{2} \sqrt{k^{2}-\Phi^{2}} \\
& j_{y}=-\frac{\hbar}{m} A_{y} F_{o}^{*} F_{o}=-\frac{\hbar}{m}|t|^{2} \Phi
\end{aligned}
$$




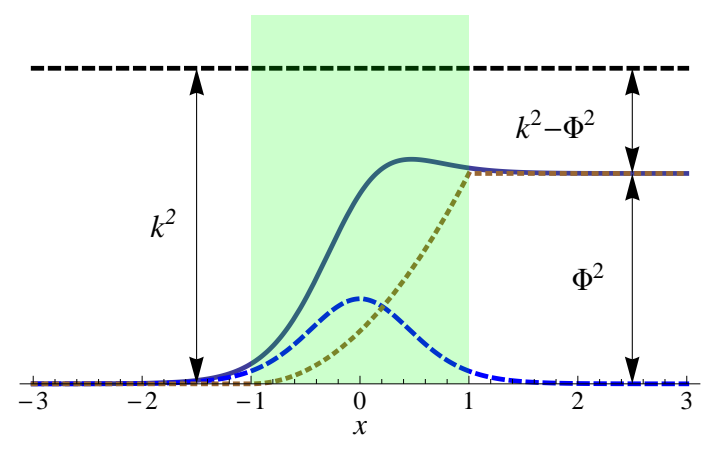

FIG. 1. (Color online) The solid blue line is a plot of Eq. 42 ) the effective potential (vertical axis not shown) as a function of $x$ (horizontal axis). The dashed blue line represents the effective scalar potential $b(x)$ given by Eq. 40). The dotted line represents the effective potential, given in Eq. (B5), for the ferromagnetic slab. The shaded region represent the slab. We have chosen the parameters, $\beta=1, L=2, B_{0}=1$.

where we made use, for $k>|\Phi|$, of the scattering boundary conditions

$$
F_{o}(x) \rightarrow t \exp \left(i \sqrt{k^{2}-\Phi^{2}} x\right) .
$$

The angle of deflection, with respect to the normal is then given by

$$
|\tan \theta|=\frac{j_{y}}{j_{x}}=\frac{|\Phi|}{\sqrt{k^{2}-\Phi^{2}}}
$$

and agrees with the result obtained in the classical description of scattering of a charged particle particle by a ferromagnetic slab (see Appendix B).

\section{Multichannel description}

The discussion presented above makes explicit use of the $\mathrm{BO}$ approximation, we now repeat the analysis using the full, and exact, multichannel description. We need to solve the coupled equations,

$$
\begin{aligned}
& -\frac{\hbar^{2}}{2 m}(\nabla-i \boldsymbol{A})^{2} \underline{F}+V \underline{F}-E \underline{F}=0 \\
& \underline{F}=\left(\begin{array}{c}
F_{c} \\
F_{o}
\end{array}\right) \\
& V=\left(\begin{array}{cc}
\Delta & 0 \\
0 & -\Delta
\end{array}\right)
\end{aligned}
$$

where $\boldsymbol{A}$ is given by Eqs. (35) and (36). The diabatic gauge amplitude $\underline{G}$ is related to $\underline{F}$ via gauge transformation Eq. 27) and it obeys

$$
\begin{aligned}
& -\frac{\hbar^{2}}{2 m} \nabla^{2} \underline{G}+W \underline{G}-E \underline{G}=0 \\
& W=U V U^{\dagger}= \\
& \left(\begin{array}{cc}
\Delta \cos (2 \Omega(x)) & e^{-i \Phi y} \Delta \sin (2 \Omega(x)) \\
e^{i \Phi y} \Delta \sin (2 \Omega(x)) & -\Delta \cos (2 \Omega(x))
\end{array}\right) .
\end{aligned}
$$

In Figure 2 we plot both the diagonal and off -diagonal components of the matrix-valued potential $W$ using the expression for $\Omega(x)$ given by Eq. (34) for $y=0$. It is somewhat more convenient to define a new amplitude so that $\underline{G}=\tilde{U} \underline{G}^{\prime}$

$$
\tilde{U}=\exp \left(-i \frac{\Phi y}{2} \sigma_{3}\right)
$$

where $\sigma_{3}$ is the diagonal Pauli matrix and $W$ is replaced by

$$
\begin{aligned}
& W \rightarrow W^{\prime}=\tilde{U}^{\dagger} W \tilde{U}= \\
& \left(\begin{array}{cc}
\Delta \cos (2 \Omega(x)) & \Delta \sin (2 \Omega(x)) \\
\Delta \sin (2 \Omega(x)) & -\Delta \cos (2 \Omega(x))
\end{array}\right)
\end{aligned}
$$

Thus

$$
\begin{aligned}
& -\frac{\hbar^{2}}{2 m}(\boldsymbol{\nabla}-i \tilde{\boldsymbol{A}})^{2} \underline{G^{\prime}}+W^{\prime} \underline{G^{\prime}}-E \underline{G^{\prime}}=0 \\
& \tilde{\boldsymbol{A}}=i \tilde{U}^{\dagger} \nabla \tilde{U}=\hat{\boldsymbol{j}}\left(\begin{array}{cc}
\frac{\Phi}{2} & 0 \\
0 & -\frac{\Phi}{2}
\end{array}\right)
\end{aligned}
$$

or, letting $\underline{G}^{\prime}=\left(\begin{array}{c}f_{1} \\ f_{2}\end{array}\right)$,

$$
\begin{aligned}
& \frac{\partial^{2} f_{1}}{\partial x^{2}}+\left(\frac{\partial}{\partial y}-i \frac{\Phi}{2}\right)^{2} f_{1}-\frac{2 m}{\hbar^{2}} \Delta \cos (2 \Omega) f_{1}+\frac{2 m}{\hbar^{2}} E f_{1}-\frac{2 m}{\hbar^{2}} \Delta \sin (2 \Omega) f_{2}=0 \\
& \frac{\partial^{2} f_{2}}{\partial x^{2}}+\left(\frac{\partial}{\partial y}+i \frac{\Phi}{2}\right)^{2} f_{2}+\frac{2 m}{\hbar^{2}} \Delta \cos (2 \Omega) f_{2}+\frac{2 m}{\hbar^{2}} E f_{2}-\frac{2 m}{\hbar^{2}} \Delta \sin (2 \Omega) f_{1}=0
\end{aligned}
$$

With the ansatz $f_{1}(x, y)=\exp \left(-i \frac{\Phi}{2} y\right) f_{1}(x)$ and $f_{2}(x, y)=\exp \left(-i \frac{\Phi}{2} y\right) f_{2}(x)$ we obtain, 

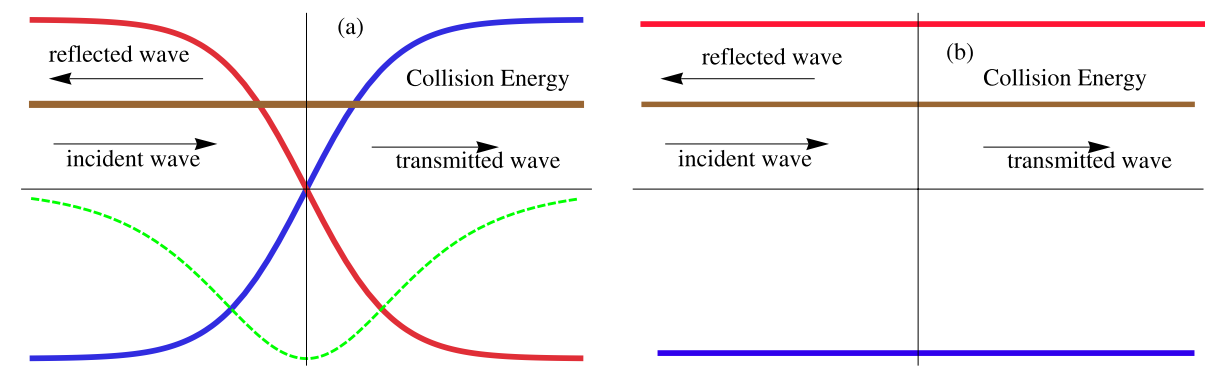

FIG. 2. (Color online) Schematic diagram of scattering setup described by Eqs. (48). (a) An incident wave in the open channel whose potential energy is given by the solid blue line. The horizontal axis represent the $x$ coordinate, and for $x<0$ the channel described by the red solid line is closed. For $x>0$ the transmitted wave is propagated on the potential surface given by the red solid line. The brown line represent the total collision energy of the system and the green dashed line represents the off-diagonal coupling between the two potential surfaces. (b) The same system now illustrated in the adiabatic picture. The blue line is the BO energy for the open channel and the red line represents the BO energy for the closed channel.

$$
\begin{aligned}
& \frac{\partial^{2} f_{1}}{\partial x^{2}}-\Phi^{2} f_{1}-\frac{2 m}{\hbar^{2}} \Delta \cos (2 \Omega) f_{1}+\frac{2 m}{\hbar^{2}} E f_{1}-\frac{2 m}{\hbar^{2}} \Delta \sin (2 \Omega) f_{2}=0 \\
& \frac{\partial^{2} f_{2}}{\partial x^{2}}+\frac{2 m}{\hbar^{2}} \Delta \cos (2 \Omega) f_{2}+\frac{2 m}{\hbar^{2}} E f_{2}-\frac{2 m}{\hbar^{2}} \Delta \sin (2 \Omega) f_{1}=0 .
\end{aligned}
$$

In the asymptotic limit $x \rightarrow-\infty \Omega \rightarrow 0$, and Eqs. (53) reduce to

$$
\begin{aligned}
& \frac{\partial^{2} f_{1}}{\partial x^{2}}-\Phi^{2} f_{1}-\frac{2 m}{\hbar^{2}} \Delta f_{1}+\frac{2 m}{\hbar^{2}} E f_{1}=0 \\
& \frac{\partial^{2} f_{2}}{\partial x^{2}}+\frac{2 m}{\hbar^{2}} \Delta f_{2}+\frac{2 m}{\hbar^{2}} E f_{2}=0
\end{aligned}
$$

whose solutions are, in this limit,

$$
\begin{aligned}
\underline{G}^{\prime} & =\exp \left(-i \frac{\Phi}{2} y\right)\left(\begin{array}{c}
r_{12} \exp (\kappa x) \\
\exp (i k x)+r_{11} \exp (-i k x)
\end{array}\right) \\
\kappa & \equiv \sqrt{\frac{2 m}{\hbar^{2}}(\Delta-E)+\Phi^{2}} \\
k & \equiv \sqrt{\frac{2 m}{\hbar^{2}}(E+\Delta)} .
\end{aligned}
$$

We have chosen this boundary condition so that

$$
\begin{gathered}
\underline{G}=\tilde{U} \underline{G}^{\prime}=\exp \left(-i \frac{\Phi y}{2} \sigma_{3}\right) \underline{G}^{\prime}= \\
\left(\begin{array}{c}
\exp (-i \Phi y) r_{12} \exp (\kappa x) \\
\exp (i k x)+r_{11} \exp (-i k x)
\end{array}\right)
\end{gathered}
$$

describes an incoming, normally incident, plane wave in the open channel. In the region $x \rightarrow \infty, \Omega \rightarrow \frac{\pi}{2}$, and Eq. 52 reduces to

$$
\begin{aligned}
& \frac{\partial^{2} f_{1}}{\partial x^{2}}-\Phi^{2} f_{1}+\frac{2 m}{\hbar^{2}} \Delta f_{1}+\frac{2 m}{\hbar^{2}} E f_{1}=0 \\
& \frac{\partial^{2} f_{2}}{\partial x^{2}}-\frac{2 m}{\hbar^{2}} \Delta f_{2}+\frac{2 m}{\hbar^{2}} E f_{2}=0
\end{aligned}
$$

whose solutions can be written in terms of scattering boundary conditions

$$
\begin{aligned}
& \underline{G}^{\prime}=\exp \left(-i \frac{\Phi}{2} y\right)\left(\begin{array}{c}
t_{12} \exp \left(i k^{\prime} x\right) \\
t_{11} \exp \left(-\kappa^{\prime} x\right)
\end{array}\right) \\
& k^{\prime}=\sqrt{k^{2}-\Phi^{2}} \quad \kappa^{\prime}=\sqrt{\frac{2 m}{\hbar^{2}}(\Delta-E)}
\end{aligned}
$$

or, in this limit,

$$
\begin{aligned}
& \underline{G}=\exp \left(-i \frac{\Phi y}{2} \sigma_{3}\right) \underline{G}^{\prime}= \\
& \left(\begin{array}{c}
\exp (-i \Phi y) t_{12} \exp \left(i k^{\prime} x\right) \\
t_{11} \exp \left(-\kappa^{\prime} x\right)
\end{array}\right) .
\end{aligned}
$$

The conserved current is given by

$$
j=\frac{i \hbar}{2 m}\left(\nabla \underline{G}^{\dagger} \underline{G}-\underline{G}^{\dagger} \nabla \underline{G}\right) .
$$

Inserting expression (56) into Eq. (60) we find that, in the asymptotic region $x \rightarrow-\infty$,

$$
\begin{aligned}
& j_{x}=\frac{i \hbar}{2 m}\left(-2 i k+2 i k\left|r_{11}\right|^{2}\right)=\frac{\hbar k}{m}\left(1-\left|r_{11}\right|^{2}\right) \\
& j_{y}=-\frac{\hbar}{2 m}\left|r_{12}\right|^{2} \exp (2 \kappa x) \rightarrow 0 .
\end{aligned}
$$

Like-wise in the region $x \rightarrow \infty$

$$
\begin{aligned}
& j_{x}=\frac{i \hbar}{2 m}\left(-2 i k^{\prime}\left|t_{12}\right|^{2}\right)=\frac{\hbar}{m} \sqrt{k^{2}-\Phi^{2}}\left|t_{12}\right|^{2} \\
& j_{y}=\frac{i \hbar}{2 m}\left(2 i \Phi\left|t_{12}\right|^{2}\right)=-\frac{\hbar}{m} \Phi\left|t_{12}\right|^{2} .
\end{aligned}
$$

We compare these solutions with those obtained in the BO approximation. According to Eq. 27,

$$
\underline{G}=U \underline{F}
$$


or

$$
\begin{aligned}
& \left(\begin{array}{c}
F_{c} \\
F_{o}
\end{array}\right)= \\
& \left(\begin{array}{c}
\exp (-i \Phi y)\left(f_{1}(x) \cos \Omega+f_{2}(x) \sin \Omega\right) \\
-f_{1}(x) \sin \Omega+f_{2}(x) \cos \Omega
\end{array}\right) .
\end{aligned}
$$

Carrying out these transformations, using Eq. (56) and Eq. (59), we find as $x \rightarrow-\infty$

$$
\underline{F}=\left(\begin{array}{c}
F_{c} \\
F_{o}
\end{array}\right)=\left(\begin{array}{c}
\exp (-i \Phi y) r_{12} \exp (\kappa x) \\
\exp (i k x)+r_{11} \exp (-i k x)
\end{array}\right)
$$

and as $x \rightarrow \infty$

$$
\left(\begin{array}{c}
F_{c} \\
F_{o}
\end{array}\right)=\left(\begin{array}{c}
t_{11} \exp \left(-\kappa^{\prime} x\right) \exp (-i \Phi y) \\
-t_{12} \exp \left(i \sqrt{k^{2}-\Phi^{2}} x\right)
\end{array}\right)
$$

Projecting out the open channel amplitude (i.e. the Born-Oppenheimer amplitude) we obtain

$$
\begin{aligned}
& F_{o} \rightarrow \exp (i k x)+r_{11} \exp (-i k x) \quad x \rightarrow-\infty \\
& F_{o} \rightarrow t_{12} \exp \left(i \sqrt{k^{2}-\Phi^{2}} x\right) \quad x \rightarrow \infty .
\end{aligned}
$$

In Fig. (3) we present the result for the transmission coefficient

$$
|T| \equiv \frac{\left|t_{12}\right|^{2} \sqrt{k^{2}-\Phi^{2}}}{k}
$$

and compare it to the expression obtained using the BO approximation where $t_{12}$ is replaced by $t$ given in Eq. (45). The numerical methodolgy, for producing the data shown in Fig. (3), is included in the Supplementary Material enclosed. The wavenumber $k$ is expressed in terms of the flux density $\Phi$ and transmission is allowed for $k>\Phi$. In the coupled channel calculations we set the closed channel parameter $\kappa^{\prime}=k$, and so for larger $k$ the BO approximation becomes valid in the limit as $\kappa^{\prime} \rightarrow \infty$. It is clear from the figure that the predictions of the $\mathrm{BO}$, or adiabatic, approximation are in very good agreement with the results obtained using the fully coupled calculations. As $\beta$ get large the transmission coefficient is suppressed since the barrier height of the repulsive potential $b(x)$, given by Eq. 40, becomes larger.

We conclude that the result of the $\mathrm{BO}$ approximation, with the presence of a vector potential that describes geometric magnetism, does indeed provide an accurate account for the dynamics and is in harmony with the predictions of the exact treatment.

\section{Open channels}

\section{Coupled equations in the diabatic picture}

According to the above discussion the amplitudes in the diabatic gauge are given by

$$
\underline{G}=\left(\begin{array}{c}
\exp (-i \Phi y) f_{1}(x) \\
f_{2}(x)
\end{array}\right)
$$

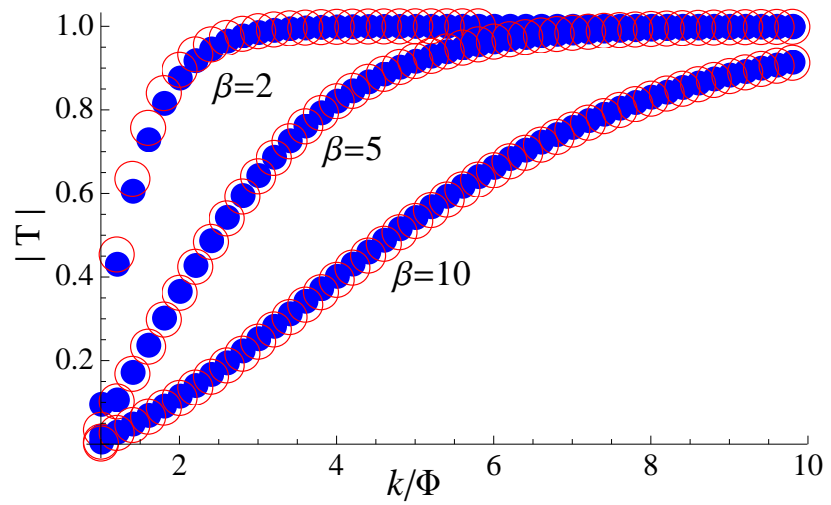

FIG. 3. (Color online) Transmission coefficient as a function of incident wavenumber $k$ expressed in units of $\Phi$ taken here to have the value of unity. The solid circles represent data obtained using the BO description, whereas the open circles denotes the transmission coefficient obtained using the coupled channel theory and given by Eq. 68

where $f_{1}(x), f_{2}(x)$ obey the coupled equations Eq. 53 . and $\Phi \equiv B_{0} L$. The expression for the conserved current is then given by

$$
\begin{aligned}
& j_{x}=\frac{\hbar}{m}\left(\operatorname{Im}\left[f_{1}^{*} \partial_{x} f_{1}\right]+\operatorname{Im}\left[f_{2}^{*} \partial_{x} f_{2}\right]\right) \\
& j_{y}=-\frac{\hbar}{m}\left|f_{1}\right|^{2} .
\end{aligned}
$$

We now evaluate the integral

$$
\mathcal{F} \equiv \int_{\mathcal{C}} d s \cdot j
$$

for contour $\mathcal{C}$ shown in Figure 4 . In that figure the current streams are generated using the procedure outlined in the supplementary material. Consider the segments labeled I,III in the diagram. The contribution to Eq. (71) from these segments are

$$
\frac{\hbar}{m} \int_{-w}^{w} d y \Phi\left(\left|f_{1}(a)\right|^{2}-\left|f_{1}(-a)\right|^{2}\right),
$$

like-wise for segment II we obtain

$$
\frac{\hbar}{m} \int_{-a}^{a} d x\left(\operatorname{Im}\left[f_{1}^{*} \partial_{x} f_{1}\right]+\operatorname{Im}\left[f_{2}^{*} \partial_{x} f_{2}\right]\right) .
$$

Since the integrand in Eq. 73 is independent of $y$ this contribution is canceled by that along segment IV. Therefore, as $|a| \rightarrow \infty$ we find

$$
\int_{C} d \boldsymbol{s} \cdot \boldsymbol{j}=\frac{\hbar}{m} 2 w \Phi\left|f_{1}(a)\right|^{2}=\frac{\hbar}{m} 2 w \Phi\left|t_{12}\right|^{2}
$$

where we made use of Eq. 599. We now consider the case where $E>\Delta+\frac{\hbar^{2} \Phi^{2}}{2 m}$ i.e. both channels in Eq. 52 
are open. As $x \rightarrow-\infty$ Eq. (56) is replaced by

$$
\underline{G} \rightarrow\left(\begin{array}{c}
\exp (-i \Phi y) r_{12} \exp \left(-i k_{2} x\right) \\
\exp (i k x)+r_{11} \exp (-i k x)
\end{array}\right)
$$

where

$$
k_{2} \equiv \sqrt{\frac{2 m}{\hbar^{2}}(E-\Delta)-\Phi^{2}}
$$

and which describes an incoming wave in channel 1 that can also reflect into channel 2. Similarly, in the region $x \rightarrow \infty$ we require

$$
\underline{G} \rightarrow\left(\begin{array}{c}
\exp (-i \Phi y) t_{12} \exp \left(i k^{\prime} x\right) \\
t_{11} \exp \left(i k_{2}^{\prime} x\right)
\end{array}\right)
$$

where

$$
\begin{aligned}
& k^{\prime}=\sqrt{k^{2}-\Phi^{2}} \\
& k_{2}^{\prime}=\sqrt{\frac{2 m}{\hbar^{2}}(E-\Delta)} .
\end{aligned}
$$

We evaluate $\int_{\mathcal{C}} d \boldsymbol{s} \cdot \boldsymbol{j}$ as above. Using Eqs. 70 we find that the contribution from segments II,IV cancel and that contributions from segments I, III give

$$
\begin{aligned}
& \mathcal{F}=\int_{\mathcal{C}} d \boldsymbol{s} \cdot \boldsymbol{j}=\frac{\hbar}{m} \Phi 2 w\left[\left|f_{1}(a)\right|^{2}-\left|f_{1}(-a)\right|^{2}\right]= \\
& \frac{\hbar}{m} \Phi 2 w\left[\left|t_{12}\right|^{2}-\left|r_{12}\right|^{2}\right] .
\end{aligned}
$$

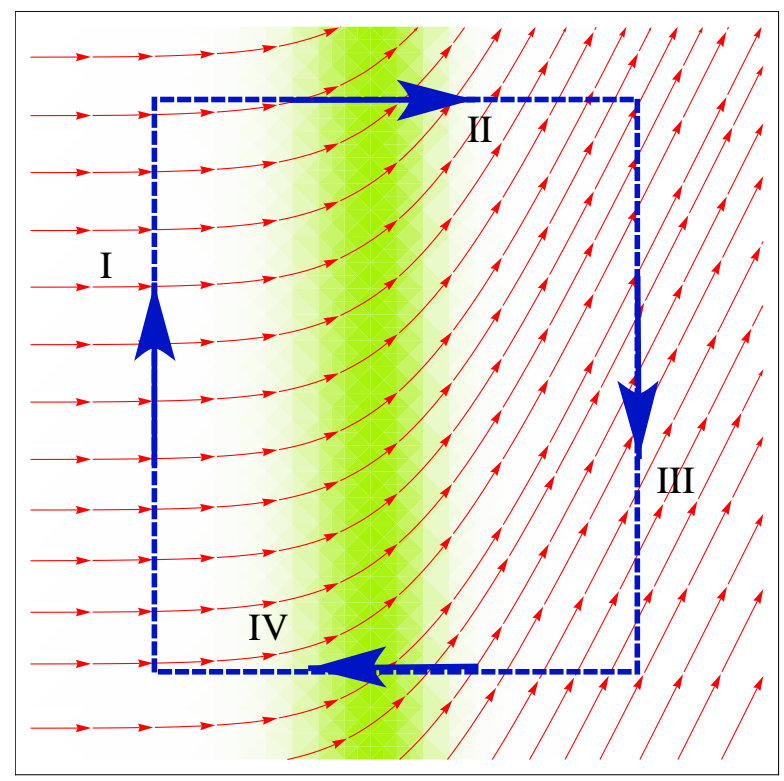

FIG. 4. (Color online) Contour plot of the probability current stream given by Eq. 60 . The incident wave is along the horizontal. The shaded green area represents region where the magnetic induction $B(x)$ given by Eq. (38) is non-negligible. Dashed blue line represents the contour $\mathcal{C}$. Segment I,III extend from $-w$ to $w$, and segments II, IV range from $-a$ to $a$. The collision energy is such that the upper adiabatic channel, shown in the diagram of Figure 2, is closed.
To summarize, we found that

$$
\begin{aligned}
& \mathcal{F}=\frac{\hbar}{m} 2 w \Phi\left|t_{12}\right|^{2} \quad E<\Delta+\frac{\hbar^{2} \Phi^{2}}{2 m} \\
& \mathcal{F}=\frac{\hbar}{m} 2 w \Phi\left[\left|t_{12}\right|^{2}-\left|r_{12}\right|^{2}\right] \quad E>\Delta+\frac{\hbar^{2} \Phi^{2}}{2 m} .
\end{aligned}
$$

\section{Coupled equations in the adiabatic picture}

Before we discuss the significance of Eq. 80 , it is useful to re-derive this result in the adiabatic representation. The gauge invariance of expression (71) follows from general principles, but it is wortwhile to investigate the role of the vector potential in its enforcement. In the adiabatic gauge the amplitudes obey Eq. (47) and lead to the conserved current

$$
\boldsymbol{j}=\frac{i \hbar}{2 m}\left(\nabla \underline{F}^{\dagger} \underline{F}-\underline{F}^{\dagger} \nabla \underline{F}+2 i \underline{F}^{\dagger} \underline{\boldsymbol{A}} \underline{F}\right)
$$

where $\underline{F}$ is given by Eq. (64). Consider first

$$
\tilde{\boldsymbol{j}} \equiv \frac{i \hbar}{2 m}\left(\nabla \underline{F}^{\dagger} \underline{F}-\underline{F}^{\dagger} \nabla \underline{F}\right) .
$$

We show below that

$$
\begin{aligned}
& \text { for } E>\Delta+\frac{\hbar^{2} \Phi^{2}}{2 m} \\
& \tilde{\mathcal{F}} \equiv \int_{\mathcal{C}} d \boldsymbol{s} \cdot \tilde{\boldsymbol{j}}=\frac{\hbar}{m} \Phi 2 w\left(\left|t_{11}\right|^{2}-\left|r_{12}\right|^{2}\right) \\
& \text { and } \\
& \tilde{\mathcal{F}}=0 \quad E<\Delta+\frac{\hbar^{2} \Phi^{2}}{2 m} .
\end{aligned}
$$

The contribution to the left-hand side of Eq. (83) from segment I is given by

$$
\begin{aligned}
& \int_{-w}^{w} d y \tilde{j}_{y}=\frac{\hbar}{m} \int_{-w}^{w} d y\left(\operatorname{Im}\left(F_{o}{ }^{*} \partial_{y} F_{o}\right)+\operatorname{Im}\left(F_{c}{ }^{*} \partial_{y} F_{c}\right)\right)= \\
& -\frac{\hbar}{m} \int_{-w}^{w} d y \Phi\left|f_{1}(-a)\right|^{2}
\end{aligned}
$$

where we have used Eq. (64) and the fact $\Omega \rightarrow 0$ as $a \rightarrow-\infty$. Like-wise, for segment III we obtain

$$
-\int_{-w}^{w} d y \tilde{j}_{y}=\frac{\hbar}{m} \int_{-w}^{w} d y \Phi\left|f_{2}(a)\right|^{2} .
$$

The contribution from segment II is

$$
\begin{aligned}
& \int_{-a}^{a} d x \tilde{j}_{x}= \\
& \frac{\hbar}{m} \int_{-a}^{a} d x\left(\operatorname{Im}\left(F_{o}{ }^{*} \partial_{x} F_{o}\right)+\operatorname{Im}\left(F_{c}{ }^{*} \partial_{x} F_{c}\right)\right),
\end{aligned}
$$

but since it is independent of $y$ it cancels with the contribution arising from segment IV. Inserting the limits for $\left|f_{1}(-a)\right|^{2}$ and $\left|f_{2}(a)\right|^{2}$, as $|a| \rightarrow \infty$, into Eq. (84) and Eq. (85) we arrive at our assertion Eq. (83). We now evaluate 


$$
\mathcal{F}_{A} \equiv-\frac{\hbar}{m} \int_{\mathcal{C}} d \boldsymbol{s} \cdot \underline{F^{\dagger}} \boldsymbol{A} \underline{F}=-\frac{\hbar}{m} \int_{\mathcal{C}} d \boldsymbol{s} \cdot\left[F_{c}{ }^{*} F_{c} \boldsymbol{A}_{11}+F_{c}{ }^{*} F_{o} \boldsymbol{A}_{12}+F_{o}{ }^{*} F_{c} \boldsymbol{A}_{21}+F_{o}{ }^{*} F_{o} \boldsymbol{A}_{22}\right]
$$

where $\boldsymbol{A}_{i j}$ are the matrix elements given in Eq. 28. The contribution from segment II,

$$
\begin{aligned}
& i \frac{\hbar}{m} \int_{-a}^{a} d x \partial_{x} \Omega\left(F_{c}{ }^{*} F_{o} \exp (-i \Phi w)-F_{o}{ }^{*} F_{c} \exp (i \Phi w)\right)= \\
& i \frac{\hbar}{m} \int_{-a}^{a} d x \partial_{x} \Omega\left(f_{1}^{*} f_{2}-f_{2}^{*} f_{1}\right),
\end{aligned}
$$

is independent of $y$ and it is canceled by the contribution from segment IV. Consider the contributions from segments I and III. First, we focus on terms that involve the off-diagonal elements,

$$
\frac{\hbar}{m} \frac{\Phi}{2} \int_{-w}^{w} d y \sin (2 \Omega)\left(F_{c}^{*} F_{o} \exp (-i \Phi y)+F_{o}{ }^{*} F_{c} \exp (i \Phi y)\right)
$$

For segments I,III, $\Omega=0, \frac{\pi}{2}$ respectively and according to Eq. 89 these contributions vanish. Finally, we consider the diagonal terms,

$$
\frac{\hbar}{m} \Phi \int_{-w}^{w} d y \sin ^{2}(\Omega)\left(F_{c}^{*} F_{c}-F_{o}^{*} F_{o}\right)
$$

whose non-vanishing contribution comes from segment III and has the value given by

$$
\mathcal{F}_{A}=\frac{\hbar}{m} \Phi \int_{-w}^{w} d y\left(\left|f_{1}(a)\right|^{2}-\left|f_{2}(a)\right|^{2}\right) .
$$

Using Eqs. 64 and 69 we obtain

$$
\begin{aligned}
& \mathcal{F}_{A}=\frac{\hbar}{m} \Phi 2 w\left(\left|t_{12}\right|^{2}-\left|t_{11}\right|^{2}\right) \quad E>\Delta+\frac{\hbar^{2} \Phi^{2}}{2 m} \\
& \mathcal{F}_{A}=\frac{\hbar}{m} \Phi 2 w\left|t_{12}\right|^{2} \quad E<\Delta+\frac{\hbar^{2} \Phi^{2}}{2 m} .
\end{aligned}
$$

Adding this contribution to that given by Eq. 83 we obtain,

$$
\begin{aligned}
& \mathcal{F}=\tilde{\mathcal{F}}+\mathcal{F}_{A}=\frac{\hbar}{m} \Phi 2 w\left(\left|t_{12}\right|^{2}-\left|r_{12}\right|^{2}\right) \quad E>\Delta+\frac{\hbar^{2} \Phi^{2}}{2 m} \\
& \mathcal{F}=\frac{\hbar}{m} \Phi 2 w\left|t_{12}\right|^{2} \quad E<\Delta+\frac{\hbar^{2} \Phi^{2}}{2 m}
\end{aligned}
$$

and which agrees with expression 80 obtained in the diabatic picture.

Equation (93) constitutes one of the main results in this paper. It expresses the scattering solutions in terms of the integral

$$
\mathcal{F} \equiv \int_{\mathcal{C}} d s \cdot j
$$

which is invariant with respect to the gauge or representation. That is, $\mathcal{F}$ has the same value in both the diabatic or adiabatic pictures. If the collision energy is such that the excited adiabatic channel is closed, then $\mathcal{F}$ does not vanish and its value is given by the second line in Eq. (93). Furthermore, in the adiabatic representation that value is determined from the contribution of the current that is proportional to $\boldsymbol{A}$. Because this result is obtained in the, exact, coupled-channel framework it does not require the invocation of the BO approximation. However, the BO approximation provides an elegant and accurate description and the appearance of a vector gauge potential in Eq. 40 is simply a consequence of the fact that the BO approximation is faithful to the requirement that $\mathcal{F}$ does not have a null value. Indeed, the arbitrary removal of the vector potential in the BO approximation compromises its efficacy.

It is important to note that the vector potential Eq. (40) and the resulting effective $\boldsymbol{B}$ field are source-less and there are no-singularities associated with it. Consider now the case where the BO approximation is no longer valid at higher collision energies where the excited adiabatic state is also open. In the high energy limit where $E>\Delta$ we can ignore $\Delta$ (in lowest order) and it is evident that in this limit

$$
\left|t_{12}\right| \rightarrow\left|r_{12}\right| \rightarrow 0
$$

and $\mathcal{F} \rightarrow 0$. Thus, at higher collision energies the scattering properties are not affected by the presence of the vector gauge potential, i.e. its influence on the system is what one would expect from that of a pure gauge. This does not preclude the fact that when working in the adiabatic picture one cannot ignore the gauge potentials when solving for the multi-channel amplitudes. An interesting 
case concerns the energy regime where both channels are open, thus voiding the BO approximation, but the limit behavior, given in Eq. (95), has not been reached. That discussion will be deferred to future investigations by the author.

In summary we have shown the necessity of including the non-trivial vector potential Eq. 40 in the BO approximation. The potential predicts a Lorentz force that determines the scattering properties of the proposed system. We illustrated how this system is an analog to that of a charged particle that is scattered by a ferromagnetic slab. We have solved the, exact, fully coupled equations in both the diabatic and adiabatic pictures and showed the fidelity of the BO approximation at low collision energies. At higher collision energies we find, in an appropriate limit, that the presence of the induced gauge potentials do not affect scattering properties. Thus the emergence of non-trivial gauge structure in the low energy limit is suggestive of phenomena that arise when a symmetry is broken 35 .

\section{E. Time-dependent treatment}

In the previous section we investigated the scattering solutions of Eq. 47) using time independent methods. In this section we exploit time-dependent methods to enhance and generalize the conclusions of the previous sections. We consider the time-dependent version of coupled equations 48 for the amplitude $\psi$,

$$
\begin{aligned}
i \hbar \frac{\partial f}{\partial t} & =-\frac{\hbar^{2}}{2 m} \nabla^{2} f+V f+V_{12} g \\
i \hbar \frac{\partial g}{\partial t} & =-\frac{\hbar^{2}}{2 m} \nabla^{2} g+V_{12}^{*} f-V g
\end{aligned}
$$

where

$$
\begin{aligned}
& \psi \equiv\left(\begin{array}{c}
f \\
g
\end{array}\right) \\
& V=\Delta \cos (2 \Omega(x)) \\
& V_{12}=\exp (-i \Phi y) \Delta \sin (2 \Omega(x)) .
\end{aligned}
$$

$\Omega(x)$ is defined in Eq. 34 and $\Phi \equiv B_{0} L$. Eqs. (96) may be solved numerically using a procedure introduced by Hermann and Fleck [36] and discussed here in the Supplementary material. In Figure $(5$ a) we provide a time series contour plot of the probability densities $|f|^{2}$ and $|g|^{2}$. At $\tau=0$ we place a Gaussian wave-packet $\psi_{0}=\left(\begin{array}{c}0 \\ g_{0}\end{array}\right)$ centered $\xi=-4.0, \eta=0$, where $(\xi, \eta)=(x / L, y / L)$ with $L$ an arbitrary length scale are dimensionless, with an initial velocity directed along the positive $\xi$ axis. In the region $(\Omega \rightarrow 0)$

$$
H_{a d}=\left(\begin{array}{cc}
\Delta & 0 \\
0 & -\Delta
\end{array}\right)
$$

and the wave packet evolves as that of a free particle until it reaches the interaction region $\xi \approx 0$. The wave-packet is illustrated by the blue contours in Figure $(57)$. The initial kinetic energy of the packet was chosen so penetration of the potential barrier, illustrated in Figure (2), is prevented. However, the packet can execute a transition into the open channel across the barrier. In other words, a transition from the $g$ to $f$ channel occurs in the region $\xi \approx 0$. This is illustrated in Figure $(5 a)$ by the red contours that represent the wave-packet, probability, contours in the $f$ channel. In addition to distortion and spreading of the packet there is a noticeable swerve in its velocity as it emerges from the interaction region. We define the adiabatic amplitudes,

$$
\begin{gathered}
\tilde{f}=f \cos \Omega(\xi)+\exp (-i \Phi \eta) \sin \Omega(\xi) g \\
\tilde{g}=g \cos \Omega(\xi)-\exp (i \Phi \eta) \sin \Omega(\xi) f
\end{gathered}
$$

and $\tilde{\psi}=\left(\begin{array}{c}\tilde{f} \\ \tilde{g}\end{array}\right)$ obeys the time-dependent analogue of Eq. 47

$$
i \hbar \frac{\partial \tilde{\psi}}{\partial t}=-\frac{\hbar^{2}}{2 m}(\boldsymbol{\nabla}-i \boldsymbol{A})^{2} \tilde{\psi}+H_{B O} \tilde{\psi},
$$

where $\boldsymbol{A}$ is a non-Abelian, pure, gauge potential. In the region $\xi \rightarrow-\infty, \Omega \rightarrow 0$ and $\tilde{g} \rightarrow g$. Likewise as $\xi \rightarrow \infty$, $\Omega \rightarrow \pi / 2$ and $\tilde{g} \rightarrow f$. This behavior is illustrated in Figure $(5 \mathrm{~b})$ where we present a $3 \mathrm{D}$ plot for the evolution of $|\tilde{g}|^{2}$. In the adiabatic picture the open channel amplitude $\tilde{g}$ evolves in a constant adiabatic potential $-\Delta$ shown in Figure (2b). As long as the collision energy is below the threshold for excitation into the upper adiabatic, or closed, channel the system evolves on a single adiabatic surface. Under such conditions the Born-Oppenheimer (BO) approximation to the solutions of Eq. (99) is appropriate. In this approximation, the projection operator $P \tilde{\psi}=\tilde{g}$, is applied on Eq. 99 to get

$$
i \hbar \frac{\partial \tilde{g}}{\partial t}=-\frac{\hbar^{2}}{2 m}(\nabla-i \tilde{\boldsymbol{A}})^{2} \tilde{g}-\left(\Delta-\frac{\hbar^{2} b(x)}{2 m}\right) \tilde{g}
$$

where $\tilde{\boldsymbol{A}}=P \boldsymbol{A} P$ is an Abelian gauge potential with non-vanishing curl and $b(x)$ is the induced scalar potential in Eq. 40. It leads to an effective magnetic induction given by Eq. (38) and mimics that incurred on a charged particle that is scattered by a ferromagnetic medium. The magnetic induction is normal to the plane of the page, and is illustrated by the green shaded area in Figure (5b). In Figure (5a) we also plot, shown by the dashed line, the trajectory for the solution of the classical equations of motion, subjected to a Lorentz force $\boldsymbol{v} \times \boldsymbol{B}$, where $|\boldsymbol{B}|$ is given by Eq. (38). Comparison of the classical path and that traced by the centers of the wave-packets shows good agreement. The deflection angle suffered by a charged particle that is normally incident on a ferromagnetic slab, of finite width, with constant magnetic induction $\boldsymbol{B}$ directed perpendicular to the plane of the page is given by Eq. 46.

In Table[1 we tabulate values of the deflection angles $\theta$, obtained by calculating the expectation values $\langle\xi(t)\rangle$, $<\eta(t)>$ for various values of incident, adiabatic, packet 


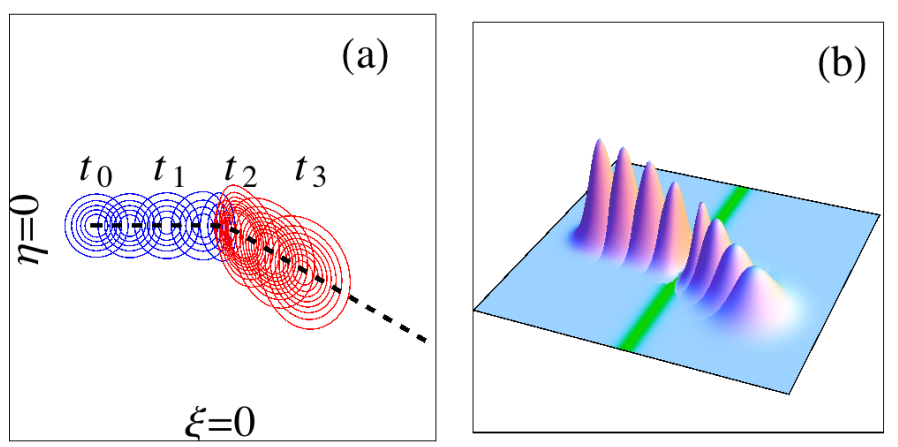

FIG. 5. (color online) (a) Circular (blue) contours represent the initial $g$ component of the wave packet probability distribution. At time $t_{2}$ the packet executes a transition into the $f$ channel, shown by the elongated (red) contours, which is energetically open in the region $\xi>0$. Subsequent to the transition the packet evolves as a free particle but with a pronounced swerve in its velocity. (b) $3 \mathrm{D}$ plot of the adiabatic gauge probability distribution $|\tilde{g}(t)|^{2}$. In both figures the deflection angle has the value $\tan \theta \approx 0.59$. In these calculations we took $k=12, \Phi=6, \Delta=200, \beta=2$ and $\tau_{0}=0, \tau_{1}=0.1, \tau_{2}=0.15, \tau_{3}=0.3$. $\xi, \eta$ range between $\mp 2 \pi$.
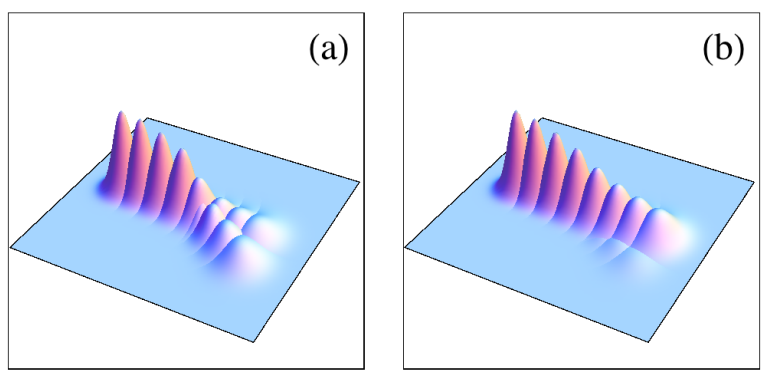

FIG. 6. (color online) Plot of probability densities $|g|^{2},|f|^{2}$ when both channels are open. The threshold energy $k_{t}^{2}=2 \Delta$. (a) $k^{2}=3 k_{t}^{2}$. (b) $k^{2}=6 k_{t}^{2}$.

wave numbers $k$ and $\Phi$. In that table we show the dependence of $\theta$ on the choice of the energy gap parameter $\Delta$. At lower collision energies, so that $k^{2}<<2 \Delta$, we find that Eq. 46 provides a good approximation for $\theta$. As the energy gap $2 \Delta$ is decreased, for a fixed value of $k$, Eq. (46) is less accurate. However, even at threshold $k^{2} \approx 2 \Delta$ there is still fairly good agreement between the calculated value and that predicted by solutions of Eq. (100). When $k^{2}>2 \Delta$ the excited adiabatic state is open and transitions from the adiabatic channel labeled $\tilde{g}$ into $\tilde{f}$ is energetically allowed. In Figure (6a) we illustrate the evolution of the amplitudes $|g(t)|^{2}$ and $|f(t)|^{2}$ for the collision energy where $2 \Delta / k^{2}=1 / 3$. The incident packet, in the $g$ channel, bifurcates when it reaches the interaction region. Because there is sufficient kinetic energy, the remainder of the initial packet proceeds along the path $\eta=0$ in the region $\xi>0$. However, a fraction of that packet makes a transition into the $f$ channel, and our calculations show that the angle of the swerve illustrated in that figure is in harmony with that obtained at the lower collision energies tabulated in Table I. Therefore there is a state-dependent spatial segregation of the initial beam,
TABLE I. Calculated values for the deflection angle $\theta$ are tabulated in the third column. The fourth column gives the values obtained using Eq. 46

\begin{tabular}{llll}
\hline \hline $2 \Delta / k^{2}$ & $\Phi / k$ & $\tan \theta$ & $\tan \theta_{c}$ \\
\hline $25 / 9$ & $1 / 2$ & 0.587 & 0.577 \\
$25 / 9$ & $1 / 4$ & 0.270 & 0.258 \\
$25 / 9$ & $1 / 12$ & 0.088 & 0.084 \\
\hline 1 & $1 / 2$ & 0.63 & 0.577 \\
1 & $1 / 4$ & 0.269 & 0.258 \\
1 & $1 / 12$ & 0.088 & 0.084 \\
\hline \hline
\end{tabular}

a hallmark of quantum control. In panel (b) of that figure we plot these probabilities for energies $2 \Delta / k^{2}=1 / 6$ and now find a small, barely noticeable, remnant of the packet in the $f$ channel. In the limit $k \rightarrow \infty$ (or $\Delta \rightarrow 0$ ) Eq. (96) allow analytic solutions and $g(t)$ simply evolves as that of a free particle. According to definition Eq. 98 ) the initial, adiabatic gauge, packet $\tilde{g}(t)$ makes a transition into the $\tilde{f}$ channel in the region $\xi>0$. This "transition" is induced by the off-diagonal gauge couplings in Eq. 99). The "transition" is simply an artifact of the adiabatic gauge (i.e. different definitions for the scattering basis in the two asymptotic regions, $\xi<0, \xi>0$ ). Because of relation (6) we conclude that $\boldsymbol{A}$ is encoded in the definition of $H_{a d}$ and since $\left[H_{B O}, \boldsymbol{A}\right] \neq 0$ gauge symmetry is explicitly broken by $H_{B O}$. Though $\boldsymbol{A}$ is trivial, in the sense of it being a pure gauge, quantum evolution selects and is sensitive to the projected $\tilde{\boldsymbol{A}}=P \boldsymbol{A P}$ nontrivial connection. In the adiabatic picture the gauge potentials are explicit, being minimally coupled to the amplitudes. As $k \rightarrow \infty$, or $\Delta \rightarrow 0$, their presence simply contributes to a multichannel, or non-Abelian, phase in the adiabatic amplitudes that has no physical import. In contrast, at lower energies the system behaves as if it has acquired a non-integrable phase factor. The effect is most pronounced when the excited adiabatic state is 
closed.

The time-dependent approach can be exploited for more complex scattering scenarios, for consider the following form of the parameter

$$
\Phi(\eta)=\frac{\eta k}{\sqrt{\eta^{2}+4 \gamma f^{2}}}
$$

where $\gamma$ is, in general, a complicated function of $\beta, k, \Delta$. Here we set it to have the constant value $\gamma=1$. Using Eq. (101) we propagate wave packets for various values of impact parameter. In Figure (7 a) we plot trajectories of the total expectation values $\langle\psi(t)|\xi| \psi(t)\rangle,\langle\psi(t)|\eta| \psi(t)\rangle$ for the various impact parameters $b$. At each impact parameter we choose identical wave-packet widths and set $k=12, \Delta=400$. The trajectories shown in that figure, by the solid red lines, demonstrate that the paths converge to a common focal point given by $f=3$. This result is gauge invariant, i.e. it can be obtained using amplitudes obtained in either diabatic or adiabatic gauges. However, the adiabatic picture provides a transparent physical description. For, in it, the system is accurately described by Eq. (100). That description includes the emergence of an effective magnetic induction $\boldsymbol{B}=\nabla \times \tilde{\boldsymbol{A}}$ whose magnitude is

$$
\frac{\pi \beta k \eta\left(8 f^{2}+\eta^{2}\right) \operatorname{sech}^{2}(\beta \xi) \cos \left(\frac{1}{2} \pi \tanh (\beta \xi)\right)}{4\left(4 f^{2}+\eta^{2}\right)^{3 / 2}}
$$

and is normal to the plane of the page. In Figure $7 \mathrm{~b}$ ) we illustrate the propagation of a coherent wave packet slab of finite width along the $\eta$ direction. After its passage through the "magnetic" lens at $\xi \approx 0$, its shape is significantly distorted. At $\tau_{1}=0.3$, where the packet describes free particle evolution, it assumes the shape of a "shark-fin" as shown in that figure. The width, along the $\eta$ direction, is significantly reduced from its value at $\tau_{0}$. A dramatic consequence of the proposed "magnetic" lensing effect. Such a lens, if realized, could find application as an "optical" component in an atom laser. In addition, consider two localized but coherent packets spatially separated at $t=0$. After passing through the lens they meet and interfere. Because of different geometric phase histories the interference pattern depends on the "magnetic" flux enclosed by the paths. One can therefore anticipate its application as a novel expression of atom interferometry.

\section{F. Aharonov-Bohm gauge potentials}

The systems in the previous sections provides illustrations of how effective gauge forces manifest in simple quantum systems, and serves as a template for more intricate systems. In the examples discussed above, we took for the internal Hamiltonian Eq. (5) where $U$ is given by Eq. (27). Such a Hamiltonian may be engineered by "dressing" a 2-level atom with a laser 13, 15. Alternatively, one can dress a spin- $1 / 2$ system that has a

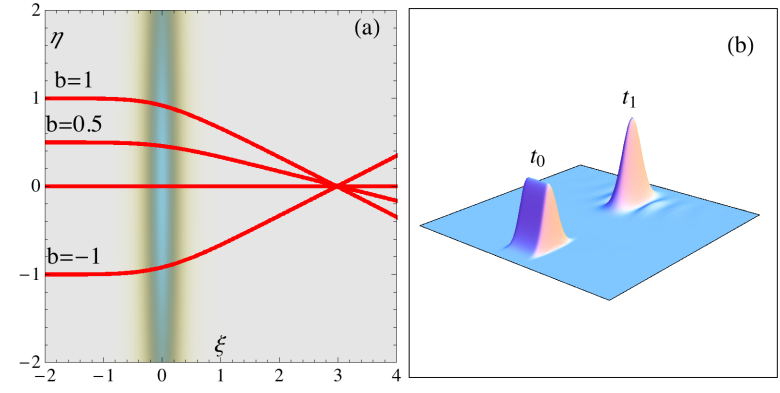

FIG. 7. (color online) (a) Trajectories of wave-packet expectation values for various values of impact parameter. The shaded region is a density plot of Eq. (102) for the "magnetic" induction. The horizontal axis gives the $\xi$ coordinate and the $\eta$ coordinate is represented by the vertical axis. (b) A wave packet slab having width $d=2$ along the $\eta$ axis at $\tau_{0}=0$ is propagated to the position shown at $\tau_{1}=0.3$.

magnetic moment with an external magnetic field, as is the case with Berry's Hamiltonian [17. Below we describe a $2 \mathrm{D}$ version of such systems. A similar, field-theoretic, analog for such a system was also proposed in Ref. 43 .

Consider that $U$ given by Eq. 27) is now replaced by

$$
U=\exp \left(-i \frac{\phi}{2} \sigma_{3}\right) \exp \left(i \frac{\pi}{4} \sigma_{1}\right) \exp \left(i \frac{\phi}{2} \sigma_{3}\right)
$$

where $\phi$ the azimuthal angle in the $x y$ plane. Hamiltonian Eq. (4) is now given by

$$
\begin{aligned}
& H=-\frac{\hbar^{2}}{2 m} \nabla_{\boldsymbol{R}}^{2}+H_{a d} \\
& H_{a d}=U(\phi) H_{B O}(\rho) U^{\dagger}(\phi) .
\end{aligned}
$$

This adiabatic Hamiltonian is identical to that of a spin $1 / 2$ particle, with a magnetic moment $\mu_{0}$, interacting with an external magnetic field in the $x y$ plane as shown in Figure (8), i.e.

$$
\begin{aligned}
& H_{a d}=\mu_{0} \boldsymbol{\sigma} \cdot \boldsymbol{B} \\
& \boldsymbol{B}=\hat{\boldsymbol{\phi}} B(\rho) .
\end{aligned}
$$

The strength of field $B(\rho)$, where $\rho$ is the distance from the origin, depends on the physical setup. For example, a wire current passing through the origin (out of the page) generates such a field with $B(\rho) \sim 1 / \rho$. For the purpose of this discussion we envision a setup in which $B(\rho)$ has the constant value $\Delta$, and then the $\mathrm{BO}$ energies are simply given by $\pm \Delta$, i.e.

$$
H_{B O}=\left(\begin{array}{cc}
\Delta & 0 \\
0 & -\Delta
\end{array}\right)
$$

and where we have set $\mu_{0}=1$.

In the adiabatic picture we again obtain Eq. 21) but with the vector potential now given by

$$
\boldsymbol{A}=\frac{\hat{\boldsymbol{\phi}}}{2 \rho}\left(\begin{array}{cc}
-1 & i e^{-i \phi} \\
-i e^{i \phi} & 1
\end{array}\right),
$$


where $\phi$ is the polar angle in a cylinder coordinate system. The diagonal scalar potential $V=H_{B O}$ contains the BO eigenvalues as its entries. Eq. (106) contains diagonal as well as the off-diagonal components that couple the two adiabatic channels. Suppose the channel with BO eigenvalue $+B(\rho)=\Delta$ is closed, i.e. the collision energy $k^{2}<\frac{2 m}{\hbar^{2}} \Delta$ as $\rho \rightarrow \infty$.

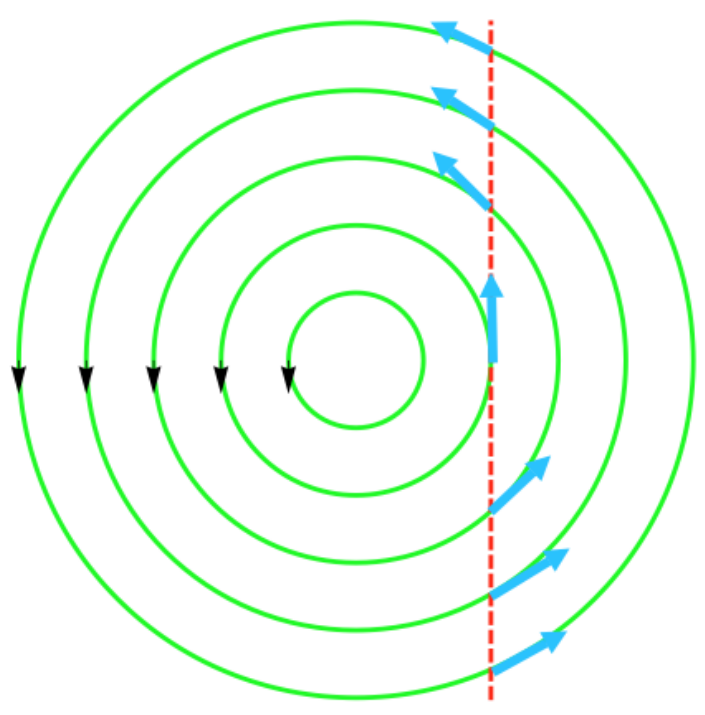

FIG. 8. (Color online) Illustration for the system described by Eq. (104. Dotted line represents a classical trajectory of the neutral atom for a given impact parameter. Green concentric circles represent the external magnetic field lines. Blue arrows represent the spin orientation of the lowest energy Zeeman level for the atom. The upper Zeeman level is closed, and so the spin of the atom is slaved to its lowest Zeeman level.

We then ignore the gauge coupling to the closed channel and project onto the open sector to obtain the approximate, one-channel, equation

$$
-\frac{\hbar^{2}}{2 m}(\boldsymbol{\nabla}-i \boldsymbol{A})^{2} F(\boldsymbol{R})+V(R) F(\boldsymbol{R})=E F(\boldsymbol{R}),
$$

where

$$
\begin{aligned}
& \boldsymbol{A}=\frac{\hat{\boldsymbol{\phi}}}{2 \rho} \\
& V(R)=-\Delta+\frac{\hbar^{2}}{8 m \rho^{2}},
\end{aligned}
$$

and the second term in Eq. 108 represents the induced scalar gauge potential[7]. This procedure is analogous to the one we carried out in the previous section for the $2 \mathrm{D}$ "magnetic" slab. There we showed that the BO projection is accurate in cases where the excited channel is closed, this conclusion also applies to this system [37. Vector potential 108 has the property

$$
\int_{C} d \boldsymbol{r} \cdot \boldsymbol{A} \neq 0
$$

where the line integral is taken along the path $C$ that encloses the origin. Because of Eq. (109) we cannot find a gauge transformation in which the vector potential is eliminated 28. Indeed this vector potential is familiar from Aharonov-Bohm scattering 38. A similar vector potential has been invoked [16 for poly-atomic systems in which conical intersections occur. It is note-worthy to mention that the adiabatic eigenstates $U(\theta) \mid \pm>$ (where $\mid \pm>$ are the eigenstates of $\left.H_{B 0}\right)$ are not multi-valued, and the $\mathrm{BO}$ eigenvalues $\pm B(\rho)$ are non-degenerate everywhere, so the analysis given in Ref. [16, 39] does not apply here. Previous studies of geometric phase effects in reactive scattering require the presence of conical intersections, as in studies of the hydrogen-exchange reaction 40. Unlike the example given in the previous sections, gauge potential (108) does posess singular behavior as $\rho \rightarrow 0$, as is evidenced by relation (109) which does not vanish in the limit as $\mathcal{C}$ encloses and contracts upon the origin. Consider the following integral

$$
W=\mathcal{P} \exp \left(i \int_{\mathcal{C}} d \boldsymbol{R} \cdot \boldsymbol{A}\right)
$$

where $\mathcal{C}$ is a path that starts at the point $\left\{\rho=R_{0}, \phi=0\right\}$ and follows a circular arc to the final point $\left\{R_{0}, \phi\right\} . \boldsymbol{A}$ is given by Eq. 106 and in order to evaluate this integral we parametrize the path $\boldsymbol{R}(t)=R_{0} \cos \omega t \hat{\boldsymbol{i}}+R_{0} \sin \omega t \hat{\boldsymbol{j}}$. According to Eq. 7]

$$
\begin{aligned}
& W(t)=T \exp \left(i \int_{0}^{t} d t^{\prime} A\left(t^{\prime}\right)\right) \\
& A\left(t^{\prime}\right)=\frac{d \boldsymbol{R}}{d t^{\prime}} \cdot \boldsymbol{A}=\frac{\omega}{2}\left(\begin{array}{cc}
-1 & i \exp (-i \omega t) \\
-i \exp (i \omega t) & 1
\end{array}\right)
\end{aligned}
$$

where $\omega t=\phi$. Now,

$$
\frac{d W}{d t}=i A(t) W
$$

which integrated, gives

$$
W(t)=\frac{1}{\sqrt{2}}\left(\begin{array}{cc}
1 & -i \exp (-i \omega t) \\
-i \exp (i \omega t) & 1
\end{array}\right) .
$$

Indeed we find, replacing $t$ with $\phi / \omega$, that $W(t)=U^{\dagger}$, given in Eq. 103). When path $\mathcal{C}$ makes a closed circuit we find $W(2 \pi / \omega)=1$ as it must. Therefore $\boldsymbol{A}$ is a pure gauge and is not singular. However its diagonal, Abelian, components $\pm \frac{\hat{\phi}}{2 \rho}$ need not be and, in this case, lead to the singular behavior evidenced by relation (109). At low collision energies, quantum dynamics effectively projects out the channel in which this manifestation of a nonintegrable phase factor emerges.

Though vector potential Eq. 108 is nontrivial it does not lead to an effective Lorentz force for $\rho \neq 0$, nevertheless its presence has a profound effect on the scattering properties of this system [37. These examples illustrate how gauge potentials arise due to the "dressing" of the atom (a spin 1/2 system) by external fields. Below we 
show how this relates to a central question of geometric magnetism, how and why do non-trivial gauge potentials arise in inter-atomic interactions? We now take our system to be that of two spin - $1 / 2$ systems that interact via a spin-spin dipolar interaction. Such systems are often realized in nature, including that for the interaction of two hydrogen atoms [21, 37]. They also serve as a template for a non-trivial two-qubit quantum gate 21]. Here the adiabatic energy splittings are generated by the internal spin-spin interaction, and the BO energy matrix is sandwiched by rotation operators similar to that given above. In an adiabatic expansion (now a four channel, or two-qubit problem) the PSS equations possess a four channel vector potential, similar to those given above and discussed in detail below.

\section{INTERNAL GAUGE POTENTIALS}

Consider Hamiltonian 105 in three spatial dimensions and with $\boldsymbol{B}$ spherically symmetric about the origin. This (or closely related to it) system has been discussed extensively in the literature 17, 24, 41. In Berry's model [17], $\boldsymbol{R}$ is treated as a classical variable, Stone quantized $\boldsymbol{R}$ and considered the full quantal solutions. Berry and Robbins 24] also studied, from a semi-classical perspective, the role of the effective Lorentz forces [5, 6. that arise in such systems. The diagonal components of $\boldsymbol{A}$ produce the effective magnetic field of a Dirac magnetic monopole [3, 42]. These gauge potentials are realized in molecular and atomic systems [3, 19] and Stone [4] suggested a "Heath-Robinson-type device", a rotating solenoid interacting with a spin $1 / 2$ particle, to serve as a physical realization.

Consider the Hamiltonian for two, interacting, spin$1 / 2$ particles [21] with magnetic moments

$$
H=-\frac{\hbar^{2}}{2 m} \nabla_{\boldsymbol{R}}^{2}+H_{a d}(\boldsymbol{R})
$$

where $\boldsymbol{R} \equiv(R, \theta, \phi)$ is the $3 \mathrm{D}$ inter-atom separation vector expressed in polar coordinates. The adiabatic Hamiltonian is given by 44

$$
\begin{aligned}
& H_{a d}=H_{0}+H_{d i p} \\
& H_{0}=\left({ }^{3} \Sigma(R)-{ }^{1} \Sigma(R)\right) \boldsymbol{S}_{a} \cdot \boldsymbol{S}_{b}+\frac{3^{3} \Sigma(R)+{ }^{1} \Sigma(R)}{4} \\
& H_{d i p}=\frac{\alpha^{2}}{R^{3}}\left[\boldsymbol{S}_{a} \cdot \boldsymbol{S}_{b}-3 \frac{\left(\boldsymbol{S}_{a} \cdot \boldsymbol{R}\right)\left(\boldsymbol{S}_{b} \cdot \boldsymbol{R}\right)}{R^{2}}\right]
\end{aligned}
$$

The scalar potentials, ${ }^{3} \Sigma(R),{ }^{1} \Sigma(R)$, are arbitrary and depend on the polarization properties of the constituents. For a structure-less system, e.g. two electrons, these scalar terms are degenerate and $H_{0}$ reduces to the longrange Coulomb interaction. In the case of two neutral spin 1/2 atoms, such as two Alkali atoms, $H_{0}$ (in atomic units) represents the isotropic electrostatic interaction between the atoms and ${ }^{3} \Sigma(R)$ and ${ }^{1} \Sigma(R)$ are the triplet and singlet $\mathrm{BO}$ energies of the electrostatic Hamiltonian respectively. $S_{i}$ are spin- $1 / 2$ operators. $H_{\text {dip }}$ is the long range component of the anisotropic magnetic interaction, $\alpha$ is a constant and for two electrons is given by the fine structure constant when atomic units are employed. We re-express 21, 45

$$
H_{a d}=U(\theta \phi) H_{B O}(R) U^{\dagger}(\theta \phi)
$$

where

$$
\begin{aligned}
& U=U_{a} \otimes U_{b} Z \\
& U_{a}=U_{b}=\exp \left(-i \sigma_{3} \phi\right) \exp \left(-i \sigma_{2} \theta\right) \exp \left(i \sigma_{3} \phi\right) \\
& Z=\left(\begin{array}{cccc}
0 & 0 & 1 & 0 \\
0 & -\frac{1}{\sqrt{2}} & 0 & -\frac{1}{\sqrt{2}} \\
0 & -\frac{1}{\sqrt{2}} & 0 & \frac{1}{\sqrt{2}} \\
1 & 0 & 0 & 0
\end{array}\right)
\end{aligned}
$$

and

$$
H_{B O}=\left(\begin{array}{cccc}
{ }^{3} \Sigma(R)-\frac{\alpha^{2}}{2 R^{3}} & 0 & 0 & 0 \\
0 & { }^{3} \Sigma(R)+\frac{\alpha^{2}}{R^{3}} & 0 & 0 \\
0 & 0 & { }^{3} \Sigma(R)-\frac{\alpha^{2}}{2 R^{3}} & 0 \\
0 & 0 & 0 & { }^{1} \Sigma(R)
\end{array}\right)
$$

is a function of the internuclear distance $R$ only. Using definition (11) we find that the induced vector potential has the block form,

$$
\begin{aligned}
\boldsymbol{A} & =\left(\begin{array}{cc}
\boldsymbol{A}_{t} & 0 \\
0 & 0
\end{array}\right) \\
\boldsymbol{A}_{t} & =\frac{1}{R}\left(\begin{array}{ccc}
\hat{\boldsymbol{\phi}} \tan \left[\frac{\theta}{2}\right] & \frac{\exp (i \phi)}{\sqrt{2}}(-i \hat{\boldsymbol{\theta}}+\hat{\boldsymbol{\phi}}) & 0 \\
\frac{\exp (-i \phi)}{\sqrt{2}}(i \hat{\boldsymbol{\theta}}+\hat{\boldsymbol{\phi}}) & 0 & \frac{\exp (i \phi)}{\sqrt{2}}(-i \hat{\boldsymbol{\theta}}+\hat{\boldsymbol{\phi}}) \\
0 & \frac{\exp (-i \phi)}{\sqrt{2}}(i \hat{\boldsymbol{\theta}}+\hat{\boldsymbol{\phi}}) & -\hat{\boldsymbol{\phi}} \tan \left[\frac{\theta}{2}\right]
\end{array}\right) .
\end{aligned}
$$


We note the following features [21, 45]

- The degenerate triplet sector of the diagonal scalar potential is split due to the inclusion of $H_{d i p}$. The states corresponding to magnetic quantum numbers $|M|=1$ contain an attractive component $-\alpha^{2} / 2 R^{3}$, whereas the $M=0$ state contains a repulsive component $\alpha^{2} / 2 R^{3}$.

- Non-trivial vector gauge potentials are induced. They contain off-diagonal components that couple the $M= \pm 1$ adiabatic channels with the $M=0$ channel. There is no direct coupling between the two $M= \pm 1$ channels, and the diagonal components are that of Dirac-magnetic monopoles of integer charges \pm 1 .

- Because the energies of the diagonal entries in $H_{B O}$ are split, a gauge transformation cannot be found in which the temporal components of the field strength tensor vanish [5]. As a consequence the gauge potentials are non-trivial in the sense described for the systems discussed in the previous sections.

When solving the scattering equations in the adiabatic gauge the induced gauge potentials, depending on the collision energy, contribute to spin changing cross sections. Though exact scattering calculations for such a system (i.e. two ground state hydrogen atoms) have been performed in the diabatic gauge 45 the question of how and in what manner the induced gauge potentials influence scattering cross sections remains to be investigated[21].

\section{SUMMARY AND DISCUSSION}

In molecular physics and atomic collision theory one is typically faced with a many-body Hamiltonian having the structure given by Eq. (4). The adiabatic Hamiltonian $H_{a d}(\boldsymbol{R}, \boldsymbol{r})$ is a function of the "fast degrees" of freedom $\mathbf{r}$ as well as $\boldsymbol{R}$, the coordinates for the positions of the atoms. Using the eigenstates of $H_{a d}$ as a basis one arrives at the set of Equations (3) in which the matrix vector gauge potential $\boldsymbol{A}$ manifests. In applications, Eq. (3) involves an infinite set of coupled equations and a truncation procedure must be invoked in order to proceed with a working theory. The remaining gauge potentials, after truncation, are non-trivial in the sense that the resulting spatial field strength tensor does not vanish $[5$ and, as a consequence, lead to effective gauge forces. They emerge in addition to the traditional Hellmann-Feynman force. We introduced and studied the gauge structure of several low-dimensional scattering systems that are solvable and where it was not necessary to employ the truncation, or $\mathrm{BO}$, approximation. For the adiabatic Hamiltonian we replaced the fast degrees of freedom with the discrete Pauli coordinates $\boldsymbol{\sigma}$,

$$
H_{a d}(\boldsymbol{R}, \boldsymbol{r}) \rightarrow H_{a d}(\boldsymbol{R}, \boldsymbol{\sigma})
$$

so that a complete set of basis functions can be used to obtain the exact solutions of the PSS equations (3). Though our choices for the adiabatic Hamiltonians varied in complexity they shared the feature that

$$
H_{a d}=U(\boldsymbol{R}) H_{B O} U^{\dagger}(\boldsymbol{R})
$$

where $U$ is a unitary operator which can be characterized by it's gauge field as given in Eq. (6). $H_{B O}$ is a diagonal matrix that contains the energy eigenvalues for $H_{a d}$, and in our discussion we were careful to choose systems in which $H_{B O}$ did not feature degeneracies at physical values for the slow parameter $\boldsymbol{R}$. The energy gap in $H_{B O}$ explictly "breaks" the symmetry inherent in the definition of $U$. The matrix structure of $H_{a d}$ depends on the particular system. For example, in our discussion relating to Figure (1) $H_{a d}$ can be "engineered" 13 by the application of a laser field on a two-level system, for the system described in Figure (8) $H_{a d}$ is generated by an external magnetic field coupled to a neutral spin-1/2 particle. In Eq. (117) $U$ is a rotation operator that characterizes the dipolar interaction Eq. 115) for two spin-1/2 particles.

For the system described by Eq. (28) we were able to solve for the scattering parameters in both the adiabatic and diabatic descriptions and showed that the quantity,

$$
\mathcal{F} \equiv \int_{\mathcal{C}} d s \cdot \boldsymbol{j}
$$

where $\boldsymbol{j}$ is a current, is invariant with respect to the choice in representation. Furthermore, for the closed loop $\mathcal{C}$ shown in Figure (4) we found that $\mathcal{F}$ does not vanish in the low-energy limit. In the adiabatic representation the non-vanishing contribution arises from the gauge potential $\boldsymbol{A}$ that define $U$. In the low-energy limit, where the excited adiabatic channel is closed, we found that the BO projection procedure serves as a good approximation for solutions obtained by the fully coupled calculation. The effective single-channel gauge potential predicts a "magnetic" field, given by Eq. (38), that leads to the appearance of an effective Lorentz force on the scattered atom. The BO amplitude which is coupled to the, nontrivial, gauge potential allows the $\mathrm{BO}$ current to satisfy the condition $\mathcal{F} \neq 0$. We compared the quantal scattering solutions of this system with that of a charged particle interacting with a ferromagnetic slab, and we found that the former predicts, according to Eq. (46), a deflection angle in harmony with that predicted by the latter. Though the multichannel gauge potential $\boldsymbol{A}$ is trivial in the sense that the spatial components of the curvature vanish, in accordance with Eq. (8), non-trivial gauge behavior manifests in the low energy limit. The adiabatic Hamiltonian does not suffer from any degeneracies, and we propose that the non-trivial gauge behavior is akin to the phenomenon of symmetry breaking 35 . This system serves as a valuable template for more complex examples, but it also highlights possible applications for geometric magnetism. For example, in section III.E we showed how these ideas can be applied toward the engineering of an effective "magnetic" lens for neutral atoms. 
We showed the relationship between systems in which dressing is carried out by external agents, such as a magnetic field for the system described by Eq. (104), or a laser field 13 for the two level system given by Eq. (27), with those in which it is accomplished by mutual inter-particle interactions. For the interacting two-qubit system give by Eqs. 116 and 117 "dressing" is accomplished by the 2-body tensor interaction Eq. (115). We argue that, regardless of the nature of the dressing agent, gauge behavior manifest in these disparate systems via the same mechanism, and we do not advocate the use different labels, e.g. fictitious, artificial, synthetic or geometric magnetism, to identify the phenomena. We propose that geometric magnetism is an appropriate terminology that underscores its universality.

\section{Appendix A: Coupled channel solution}

We compare the solution of the approximate adiabatic solution Eq. (24) to that obtained by solving the coupled equations (21). For the sake of economy in notation we set $\hbar=1$ in this appendix. In region $x>0$ we take the ansatz

$$
\underline{F}_{a d}=\left(\begin{array}{c}
F_{c} \\
F_{o}
\end{array}\right)=\exp (i \Omega x)\left(\begin{array}{c}
c_{c} \\
c_{o}
\end{array}\right) .
$$

Inserting this ansatz into Eq. 21 we obtain a set of homogeneous linear equations for the coefficients $c_{o}, c_{c}$ whose non-trivial solutions require that $\Omega$ obey the eigenvalue equations

$$
\begin{aligned}
& -\Omega^{2}-A_{0}^{2}-A_{1}^{2}+2 m E \mp \\
& \sqrt{\left(2 m \Delta-2 A_{0} \Omega\right)^{2}+\left(2 A_{1} \Omega\right)^{2}}=0 .
\end{aligned}
$$

Analytic expressions for the eigenvalues can be gleaned using the formula of Ferraria [4] for the roots of the quartic equation. An outline for calculating the solutions is given in the Supplement. A simpler and transparent expression that is valid in the, adiabatic, limit where $m \rightarrow \infty$ is sufficient for our purposes. In this limit the roots are given by

$$
\begin{aligned}
& \Omega_{c} \approx A_{0} \pm 2 i \sqrt{m \Delta} \\
& \Omega_{o} \approx-A_{0} \pm \sqrt{k^{2}-A_{1}^{2}} .
\end{aligned}
$$

where we set $E=-\Delta+\frac{\hbar^{2} k^{2}}{2 m}$ and require that $\frac{\hbar^{2} k^{2}}{2 m}<2 \Delta$ i.e. the collision energy is much smaller than the energy defect between the two BO potentials. We then obtain the following, approximate, solutions to Eq. (21), for $x>0$,

$$
\underline{F}_{c}=\exp \left(i A_{0} x\right) \exp ( \pm \sqrt{4 m \Delta} x) \times\left(\begin{array}{c} 
\pm i \\
\frac{A_{1}}{\sqrt{m \Delta}}
\end{array}\right)
$$

and

$$
\underline{F}_{o}=\exp \left(-i A_{0} x\right) \exp \left( \pm i \sqrt{k^{2}-A_{1}^{2}} x\right)\left(\begin{array}{l}
0 \\
1
\end{array}\right) .
$$

In the region $x<0$ we obtain

$$
\underline{F}=\left(\begin{array}{c}
S_{c o} \exp (\sqrt{4 m \Delta} x) \\
\exp (i k(x-L))+R \exp (-i k(x-L))
\end{array}\right)
$$

and solve for the open channel reflection coefficient $R$ by requiring the solution at $x>0$ to match the boundary condition at $x=L$, and then matching it with the asymptotic solutions at $x=0$. However, care must be taken when matching the derivatives of the adiabatic amplitudes since the vector potential $A$ is discontinuous across the boundary. We require the diabatic amplitudes given in Eq. 18 and their derivatives to be continuous across the boundary. However since the adiabatic amplitudes are related to them according to Eq. 20 we require that

$$
\underline{F}_{a d>}^{\prime}-\underline{F}_{a d<}^{\prime}=i A \underline{F}_{a d}(0)
$$

where $\underline{F}_{a d>}^{\prime}, \underline{F}_{a d<}^{\prime}$ are the derivatives of the amplitudes in the regions $x=0 \pm \epsilon$ respectively, and $\underline{F}_{a d}(0)$ is the value of the amplitude at $x=0$. Matching the amplitudes and enforcing condition Eq. A6 we obtain, as $k \rightarrow 0$

$$
R=-1+2 i k\left(L-\frac{\tanh \left(A_{1} L\right)}{A_{1}}\right)+\mathcal{O}\left(k^{2}\right) .
$$

In the limit where $\Delta=0$ the exact eigenvalues $\Omega=$ $\pm k \pm \sqrt{A_{0}^{2}+A_{1}^{2}}$, allow simple analytic expressions for solutions of Eq. (21). Though the eigensolutions are non-trivial (see Supplementary material) in the region $x>0$, carrying out the procedure that resulted in Eq. A7) leads to $R=-1$ as it must.

\section{Appendix B: Scattering by a 2D ferromagnetic strip}

We consider the scattering of a charged particle (here we set $\frac{q}{\hbar c}=1$ ) by a $2 \mathrm{D}$ ferromagnetic slab as shown in Figure 9. The particle with energy $E$ is traveling to the right, along the $x$-direction, and impacts the slab, in the normal direction, of thickness $L$. We consider the Schrodinger equation

$$
-\frac{\hbar^{2}}{2 m}(\boldsymbol{\nabla}-i \boldsymbol{A})^{2} \psi=E \psi
$$

The vector potential $\boldsymbol{A}=A \hat{\boldsymbol{j}}$ is given by

$$
\begin{aligned}
& A=B_{0} \theta(x+L / 2)(x+L / 2) \theta(L / 2-x)+ \\
& \theta(x-L / 2) L B_{0}
\end{aligned}
$$

and

$$
\begin{aligned}
& \boldsymbol{B}=\hat{\boldsymbol{z}}\left(\frac{\partial A_{y}}{\partial x}-\frac{\partial A_{x}}{\partial y}\right)= \\
& \hat{\boldsymbol{z}} B_{0} \theta(x+L / 2) \theta(L / 2-x) .
\end{aligned}
$$

We assume the particle approaches from the left (for $x<$ $-L / 2$ ) with the incident wave boundary condition,

$$
\psi=\exp (i k x)+r \exp (-i k x)
$$


where $k=\sqrt{\frac{2 m}{\hbar^{2}} E}$ and $r$ is the reflection coefficient. In the region $-L / 2<x<L / 2$ the Schrodinger equation becomes,

$$
\frac{\partial^{2} \psi}{\partial x^{2}}-B_{0}^{2}(x+L / 2)^{2} \psi+k^{2} \psi=0
$$

whose solutions are,

$$
c_{1} D_{\nu_{1}}\left(\sqrt{B_{0} / 2}(L+2 x)\right)+c_{2} D_{\nu_{2}}\left(i \sqrt{B_{0} / 2}(L+2 x)\right) .
$$

where

$$
\begin{aligned}
& \nu_{1} \equiv \frac{k^{2}}{2 B_{0}}-\frac{1}{2} \\
& \nu_{2} \equiv-\frac{k^{2}}{2 B_{0}}-\frac{1}{2}
\end{aligned}
$$

and $D_{\nu}(x)$ are the Parabolic Cylinder Functions [47]. For $x>L / 2$ the amplitudes satisfy,

$$
\begin{aligned}
& \frac{\partial^{2} \psi}{\partial x^{2}}-\Phi^{2} \psi+k^{2} \psi=0 \\
& \Phi=B_{0} L
\end{aligned}
$$

and whose form is determined by the scattering boundary conditions

$$
\begin{array}{rr}
\psi(x)=t \exp \left(i \sqrt{k^{2}-\Phi^{2}} x\right) & k>|\Phi| \\
\psi(x)=t \exp \left(-\sqrt{\left|k^{2}-\Phi^{2}\right|} x\right) & k<|\Phi|
\end{array}
$$

\section{Reflection and transmission coefficients}

We now solve for reflection and transmission coefficients. We take the logarithmic derivative, at $x=L / 2$, of the internal solution Eq. (B6) and match it with that obtained using the asymptotic boundary condition Eq. (B8) resulting in the ratio,

$$
\begin{aligned}
& \gamma \equiv c_{2} / c_{1}= \\
& \frac{D_{\nu_{1}}^{\prime}\left(\sqrt{2 B_{0}} L\right)-i \sqrt{k^{2}-\Phi^{2}} D_{\nu_{1}}\left(\sqrt{2 B_{0}} L\right)}{-D_{\nu_{2}}^{\prime}\left(i \sqrt{2 B_{0}} L\right)+i \sqrt{k^{2}-\Phi^{2}} D_{\nu_{2}}\left(i \sqrt{2 B_{0}} L\right)} .
\end{aligned}
$$

Taking the incident wave and matching it, and its derivative, with the internal solution we obtain

$$
\begin{aligned}
& r=\exp (-i L k) \frac{k+i y}{k-i y} \\
& y=\frac{D_{\nu_{1}}^{\prime}+\gamma D_{\nu_{2}}^{\prime}}{D_{\nu_{1}}+\gamma D_{\nu_{2}}}
\end{aligned}
$$

and

$$
\begin{aligned}
& t=2 i k \exp \left(-i \frac{L k}{2}\right) \exp \left(-\frac{i L}{2} \sqrt{k^{2}-\Phi^{2}}\right) \times \\
& \frac{\left(D_{\nu_{1}}\left(\sqrt{2 B_{0}} L\right)+\gamma D_{\nu_{2}}\left(i \sqrt{2 B_{0}} L\right)\right)}{D_{\nu_{1}}^{\prime}+\gamma D_{\nu_{2}}^{\prime}+i k\left(D_{\nu_{1}}+\gamma D_{\nu_{2}}\right)}
\end{aligned}
$$

where we used the shorthand $D_{\nu} \equiv D_{\nu}(0)$.

Consider the limit $L \rightarrow 0$ as the flux density $\Phi \equiv B_{0} L$ stays constant. We find, in this limit,

$$
\gamma \rightarrow i+\mathcal{O}(\sqrt{L})
$$

as

$$
y \rightarrow i \sqrt{k^{2}-\Phi^{2}} .
$$

Thus

$$
\begin{aligned}
& r \rightarrow \frac{k-\sqrt{k^{2}-\Phi^{2}}}{k+\sqrt{k^{2}-\Phi^{2}}} \\
& t=1+r
\end{aligned}
$$

and are the scattering parameters obtained for a system in which a particle is incident on a step function potential of height $\Phi^{2}$ at $x>0$.

\section{Conserved currents}

For the Schrodinger equation

$$
i \hbar \frac{\partial \psi}{\partial t}=-\frac{\hbar^{2}}{2 m}(\boldsymbol{\nabla}-i \boldsymbol{A})^{2} \psi+\frac{\hbar^{2} k^{2}}{2 m} \psi
$$

the current density is given by

$$
\boldsymbol{j}=\frac{i \hbar}{2 m}\left(\psi \boldsymbol{\nabla} \psi^{*}-\psi^{*} \boldsymbol{\nabla} \psi+2 i \boldsymbol{A} \psi^{*} \psi\right) .
$$

Inserting expression Eq. (??) into Eq. (B16) we obtain

$$
j_{x}=\frac{\hbar k}{m}\left(1-|r|^{2}\right)
$$

which can be expressed as incident $j_{i n}=\frac{\hbar k}{m}$ and reflected $j_{r}=-\frac{\hbar k}{m}|r|^{2}$ currents, respectively. In the region $x>$ $L / 2$ we find

$$
\begin{aligned}
j_{x} & =\frac{\hbar}{m}|t|^{2} \sqrt{k^{2}-\Phi^{2}} \\
j_{y} & =-\frac{\hbar}{m}|t|^{2} \Phi .
\end{aligned}
$$

where we have used Eqs. (B2) and (B8). Thus the transmitted current makes an angle $\theta$ with respect to the normal given

$$
\tan \theta \equiv \frac{j_{y}}{j_{x}}=-\frac{\Phi}{\sqrt{k^{2}-\Phi^{2}}}
$$

where $\Phi=B_{0} L$, and agrees with the classical angle of deflection.

\section{ACKNOWLEDGMENTS}

This work was supported, in part, by NSF grant PHY0758140. I wish to thank an anonymous reviewer for helpful suggestions. 


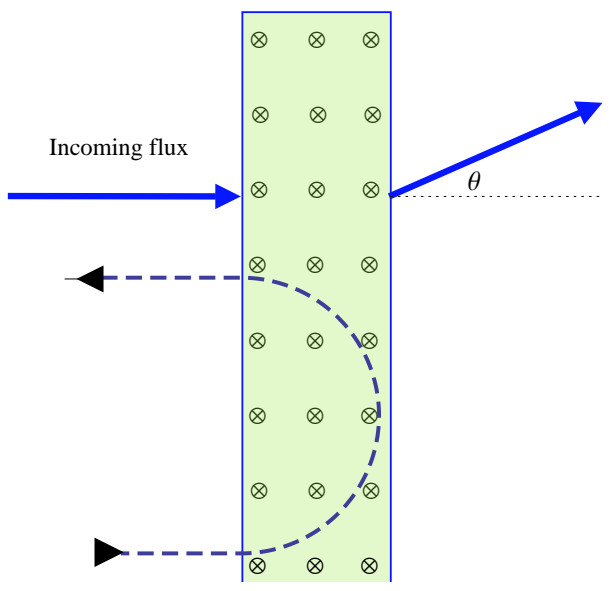

FIG. 9. (Color online) Normal incidence of a charged particle on a ferromagnetic slab of width $L$. The magnetic field $\boldsymbol{B}$ is directed into the page and the dashed line depicts a typical classical trajectory. In this figure the classical trajectory describes a particle that does not have the required velocity to overcome the barrier shown in Fig. 1

[1] R. P. Feynman, Phys. Rev. 56, 340 (1939).

[2] A. Dalgarno and R. McCarroll, Royal Society of London Proceedings Series A 237, 383 (1956)

[3] J. Moody, A. Shapere, and F. Wilczek, Phys. Rev. Lett. 56, 893 (1986).

[4] A. Shapere and F. Wilczek, Geometric Phases in Physics (World Scientific Publishing Company, 1989).

[5] B. Zygelman, Phys. Lett. A 125, 476 (1987).

[6] M. V. Berry, in Geometric Phases in Physics, edited by A. Shapere and F. Wilczek (World Scientific Publishing Company, 1989) p. 1.

[7] B. Zygelman, Physical Review Letters 64, 256 (1990). We correct a typo in Eq. (7) of that paper. A factor of $R$ should be inserted in the denominator of that expression

[8] K. Y. Bliokh and Y. P. Bliokh, Annals of Physics 319, 13 (2005).

[9] Y. Lin, R. L. Compton, A. R. Perry, W. D. Phillips, J. V. Porto, and I. B. Spielman, Physical Review Letters 102, 130401 (2009)

[10] Y. Lin, R. L. Compton, K. Jiménez-García, J. V. Porto, and I. B. Spielman, Nature 462, 628 (2009)

[11] J. Simon, W. S. Bakr, R. Ma, M. E. Tai, P. M. Preiss, and M. Greiner, Nature 472, 307 (2011).

[12] I. B. Spielman, Nature 472, 301 (2011).

[13] J. Dalibard, F. Gerbier, G. Juzeliūnas, and P. Öhberg, Reviews of Modern Physics 83, 1523 (2011).

[14] G. Juzeliūnas, J. Ruseckas, and J. Dalibard, Phys. Rev. A. 81, 053403 (2010).

[15] B. Juliá-Díaz, D. Dagnino, K. J. Günter, T. Graß, N. Barberán, M. Lewenstein, and J. Dalibard, Phys. Rev. A 84, 053605 (2011).

[16] C. A. Mead and G. D. Truhlar, The Journal of Chemical Physics 70, 2284 (1979)

[17] M. V. Berry, Proc. R. Soc. Lond. A 392, 45 (1984).

[18] F. Wilczek and A. Zee, Phys. Rev. Lett. 52, 2111 (1984).
[19] B. Zygelman, D. L. Cooper, M. J. Ford, A. Dalgarno, J. Gerratt, and M. Raimondi, Phys. Rev. A 46, 3846 (1992)

[20] B. Zygelman, A. Dalgarno, and R. Sharma, Phys. Rev. A 49, 2587 (1994).

[21] B. Zygelman, in Proceedings of the Dalgarno Celebratory Symposium: Contributions to Atomic, Molecular, and Optical Physics, Astrophysics, and Atmospheric Physics, edited by J. F. Babb, K. Kirby, and H. Sadeghpour (World Scientific Publishing Company, 2009).

[22] N. F. Mott and H. S. W. Massey, The Theory of Atomic Collisions, 3rd ed. (Oxford, 1965) p. 428.

[23] F. T. Smith, Phys. Rev. 179, 111 (1969)

[24] M. V. Berry and J. M. Robbins, Proc. R. Soc. Lond. A442, 641 (1992).

[25] M. V. Berry, Proc. R. Soc. Lond. A 452, 1207 (1996).

[26] M. V. Berry and P. Shukla, J. Phys. A: Math. Theor. 43, 045102 (2010).

[27] Robert L. Karp, Freydoon Mansouri, Jung S. Rno, Turk. J. Phy. 24, 365, (2000).

[28] T. T. Wu and C. N. Yang, Nuclear Physics B 107, 365 (1976)

[29] W. Kołos and L. Wolniewicz, Rev. Mod. Phys. 35, 473 (1963)

[30] M. Marinescu and A. Dalgarno, Phys. Rev. A 57, 1821 (1998).

[31] P. Gosselin and H. Mohrbach, J. Phys. A: Math. Theor. 43, 354025 (2010).

[32] H. Feshbach, Annals of Physics 5, 357 (1958)

[33] B. Zygelman, Unpublished (2011).

[34] G. Juzeliūnas, J. Ruseckas, P. Öhberg, and M. Fleischhauer, Phys. Rev. A. 73, 025602 (2006)

[35] Y. Nambu and G. Jona-Lasino, Physical Review 122, 345 (1961).

[36] M. R. Hermann and J. A. Fleck, Jr., Phys. Rev. A 38, $6000(1988)$. 
[37] B. Zygelman, APS Meeting Abstracts , 1027 (2010).

[38] A. Aharonov and D. Bohm, Phys. Rev. 115, 485 (1959).

[39] C. A. Mead, Rev. Mod. Phys. 64, 51 (1992)

[40] J. C. Juanes-Marcos, S. C. Althorpe, and E. Wrede, Science 309, 1227 (2005)

[41] M. Stone, Phys. Rev. D 33, 1191 (1986)

[42] P. A. M. Dirac, Royal Society of London Proceedings Series A 133, 60 (1931).
[43] John March-Russel, John Preskill, Frank Wilczek, Phys. Rev. Lett. 68, 2567 (1992).

[44] B. Zygelman, APS Meeting Abstracts , 1173 (2010).

[45] B. Zygelman, Phys. Rev. A. 81, 032506 (2010)

[46] W. Carpenter, Mathematics Magazine 39, 28 (1966).

[47] J. C. P. Miller, in Handbook of Mathematical Functions, edited by M. Abramowitz and I. A. Stegun (Dover Publications, Inc. New York, 1972) p. 685. 